\title{
Joint structural annotation of small molecules using liquid chromatography retention order and tandem mass spectrometry data
}

\author{
Eric Bach ${ }^{1, *}$, Emma L. Schymanski ${ }^{2}$ and Juho Rousu ${ }^{1, *}$ \\ ${ }^{1}$ Department of Computer Science, Aalto University, Espoo, Finland \\ ${ }^{2}$ Luxembourg Centre for Systems Biomedicine (LCSB), University of Luxembourg, \\ 6 avenue du Swing, L-4367 Belvaux, Luxembourg. \\ * Corresponding author: firstname.lastname@aalto.fi
}

\begin{abstract}
We present LC-MS ${ }^{2}$ Struct, a machine learning framework for structural annotation of small molecule data arising from liquid chromatography-tandem mass spectrometry (LC$\mathrm{MS}^{2}$ ) measurements. LC-MS ${ }^{2}$ Struct predicts the annotations for a set of mass spectrometry features in a sample, using the ions' observed retention orders and the output of state-ofthe-art $\mathrm{MS}^{2}$ scorers. LC-MS ${ }^{2}$ Struct is based on a novel structured prediction model trained to benefit from dependencies between retention times and the mass spectral features for an improved annotation accuracy.

We demonstrate the benefit of LC-MS ${ }^{2}$ Struct on a comprehensive dataset containing reference $\mathrm{MS}^{2}$ spectra and retention times of 4327 molecules from MassBank, measured using a variety of LC conditions. We show that LC-MS ${ }^{2}$ Struct obtains significantly higher annotation accuracy than methods based on retention time prediction. Furthermore, LC-MS ${ }^{2}$ Struct improves the annotation accuracy of state-of-the-art $\mathrm{MS}^{2}$ scorers by up to 66.1 percent and even up to 95.9 percent when predicting stereochemical variants of small molecules.
\end{abstract}

\section{Introduction}

Structural annotation of small molecules in biological samples is a challenging task and a bottleneck in various research fields including biomedicine, biotechnology, drug discovery and environmental sciences. Samples in untargeted metabolomics studies typically contain thousands of different molecules, most of which remain unidentified 1-3. Liquid chromatography (LC) tandem mass spectrometry $\left(\mathrm{LC}-\mathrm{MS}^{2}\right)$ is one of the most widely used analysis platforms [4], as it allows for highthroughput screening, has high sensitivity and is applicable to a wide range of molecules. Briefly, in LC-MS ${ }^{2}$, molecules are first separated by their different physicochemical interactions between the mobile and stationary phase of the LC, resulting in retention time (RT) differences. Subsequently, separation happens according to their mass-to-charge ratio $(m / z)$ in a mass analyzer $\left(\mathrm{MS}^{1}\right)$. Finally, the molecular ions are isolated and fragmented in the tandem mass spectrometer $\left(\mathrm{MS}^{2}\right)$, typically using a narrow mass window. For each ion, the recorded fragments and their intensities constitute what is called the $\mathrm{MS}^{2}$ spectrum. In an untargeted LC-MS ${ }^{2}$ workflow, large sets of MS features $\left(\mathrm{MS}^{1}, \mathrm{MS}^{2}, \mathrm{RT}\right)$, arise from a single sample. The goal in structural annotation is to associate each feature with a candidate molecular structure, for further downstream interpretation.

In recent years, many powerful methods [5, 6] to predict molecular structure annotations for $\mathrm{MS}^{2}$ spectra have been developed $7 \cdot-18$. In general, these methods find candidate molecular structures potentially associated with the MS feature, for example, by querying molecules with a certain mass from a structure database (DB) such as HMDB [19 or PubChem 20] and, subsequently, compute a matching score between each candidate and the $\mathrm{MS}^{2}$ spectrum. The highest scoring candidate is typically considered as the structure annotation of a given $\mathrm{MS}^{2}$. However, 
even the best-of-class methods only reach an annotation accuracy of around 40\% [17 in evaluation when searching large candidate sets like PubChem, and therefore, in practice, a ranked list of molecular structures is provided to the user (e.g. top 20 structures).

Even though readily available in all $\mathrm{LC}-\mathrm{MS}^{2}$ pipelines and recognized as valuable information 21, 22, RT remains underutilized in automated approaches for structure annotation based on $\mathrm{MS}^{2}$. For example, only one of the above mentioned tools provides functionality to use the RT information, namely MetFrag [11]. An explaining factor for this is that RT not only depends on the molecular structure, but also the LC conditions (e.g., mobile phase composition, column pressure, etc.) 23, 24. Thus, a molecule generally has different RTs under different LC conditions and in different laboratories 24]. Typically, the RT information is used as post-processing for candidate lists, e.g., by comparing measured and reference standard RTs 3. 24]. This approach, however, is limited by the availability of experimentally determined RTs of reference standards. RT prediction models 25, 24, on the other hand, allow to predict RTs solely based on the candidates' molecular structure and have been successfully applied to aid structure annotation 26 29. However, such prediction models generally have to be calibrated to the target LC configuration 3]. Calibration requires at least some amount of target $\mathrm{LC}$ reference $\mathrm{RT}$ data to be available [21, 30, 29].

Recently, the idea of predicting retention orders (RO), i.e., the order in which two molecules elute from the LC column, has been explored 31 34. ROs are largely preserved within a family of LC systems (e.g. reversed phase or HILIC). Therefore, RO predictors can be trained using a diverse set of RT reference datasets and applied to out-of-dataset LC setups with high accuracy 31]. Integration of $\mathrm{RO}$ and $\mathrm{MS}^{2}$ based scores using probabilistic graphical models was shown to improve the annotation performance in LC-MS ${ }^{2}$ experiments 34 .

In this study we set out to provide a new perspective on jointly using $\mathrm{MS}^{2}$ and $\mathrm{RO}$ information for the structure annotation of $\mathrm{LC}_{-} \mathrm{MS}^{2}$ data. For that, we present a novel machine learning framework called LC-MS ${ }^{2}$ Struct, which learns to optimally combine the $\mathrm{MS}^{2}$ and RO information for the accurate annotation of a sequence of MS features. LC-MS ${ }^{2}$ Struct relies on the Structured Support Vector Machine (SSVM) [35] and Max-margin Markov Network [36] frameworks. In contrast to the previous work by Bach et al. 34, our framework does not require a separately learned RO prediction model. Instead, it optimizes the SSVM parameters such that the score margin between correct and any other sequence of annotations is maximized, subject to a graphical model representing the pairwise ROs as edges and the candidate sets of molecular structures for each MS feature as candidate node labels. That means that LC-MS ${ }^{2}$ Struct learns to optimally use the RO information in an LC-MS ${ }^{2}$ experiment. We trained LC-MS ${ }^{2}$ Struct on all available reversed phase LC data from MassBank (MB) 37, which we processed to extract ground-truth annotated $\left(\mathrm{MS}^{2}, \mathrm{RT}\right)$-tuples covering a diverse set of LC and MS configurations. In our experiments we evaluate LC-MS ${ }^{2}$ Struct across all subsets of homogeneous LC-MS ${ }^{2}$ configurations and compare it with three other previously proposed approaches: RT filtering, $\log P$ predictions [1], and RO predictions 34. Our framework can be combined with any $\mathrm{MS}^{2}$ scorer and applied to new LC-MS data, including new LC conditions without re-training, and is demonstrated below with CFM-ID 9, 18, MetFrag [1] and SIRIUS [8, 17.

\section{Overview of LC-MS ${ }^{2}$ Struct}

In this section we discuss the main components of LC-MS ${ }^{2}$ Struct, which are also illustrated in Figure 1. Further details can be found in the Methods section.

Input and output. As input we consider a typical data setting present in an untargeted LC$\mathrm{MS}^{2}$ based experiments, after pre-processing such as chromatographic peak picking and alignment (Figure 1a). Such data comprises a sequence of MS features, here indexed by $\sigma$. Each feature consists of $\mathrm{MS}^{1}$ information (e.g. mass, adduct and isotope pattern), LC retention time (RT) $t_{\sigma}$ and an $\mathrm{MS}^{2}$ spectrum $x_{\sigma}$. We assume that a set of candidate molecules $\mathcal{C}_{\sigma}$ is associated with each MS feature $\sigma$. Such a set can be, for example, generated from a structure database (e.g. PubChem [20, ChemSpider 38] or PubChemLite [39]) based on the ion's mass, a suspect list, or an in silico 
molecule generator (e.g. SmiLib v2.0 [40, 41]). We furthermore require that for $\mathrm{MS}^{2}$ spectrum $x_{\sigma}$, a matching score $\theta\left(x_{\sigma}, m\right)$ with its candidates $m \in \mathcal{C}_{\sigma}$ is pre-computed using an in silico tool, such as CFM-ID 9, 18, MetFrag 11] or SIRIUS 8, 17. LC-MS ${ }^{2}$ Struct predicts a score for MS feature $\sigma$ and each associated candidate $m \in \mathcal{C}_{\sigma}$ based sequence of spectra $\mathbf{x}=\left(x_{\sigma}\right)_{\sigma=1}^{L}$, of length $L$, and the ROs derived from the observed RTs $\mathbf{t}=\left(t_{\sigma}\right)_{\sigma=1}^{L}$. These scores are used to rank the molecular candidates associated with the MS features (Figure $1 \mathbf{b}$ ).

Candidate ranking using max-marginals. We define a fully connected graph $G=(V, E)$ capturing the MS features and modelling their dependencies (Figure 1 c). Each node $\sigma \in V$ corresponds to a MS feature, and is associated with the pre-computed $\mathrm{MS}^{2}$ matching scores $\theta\left(x_{\sigma}, m\right)$ between the $\mathrm{MS}^{2}$ spectrum $x_{\sigma}$ and all molecular candidates $m \in \mathcal{C}_{\sigma}$. The graph $G$ contains an edge $(\sigma, \tau) \in E$ for each MS feature pair. A scoring function $F$ is defined predicting a compatibility score between a sequence of molecular structure assignments $\mathbf{y}=\left(y_{\sigma}\right)_{\sigma=1}^{L}$ in the label-space $\Sigma=\mathcal{C}_{1} \times \ldots \times \mathcal{C}_{L}$ and the observed data:

$$
F(\mathbf{y} \mid \mathbf{x}, \mathbf{t}, \mathbf{w}, G)=\underbrace{\frac{1}{|V|} \sum_{\sigma \in V} \theta\left(x_{\sigma}, y_{\sigma}\right)}_{\text {Node scores: } \text { MS }^{2} \text { information }}+\underbrace{\frac{1}{|E|} \sum_{(\sigma, \tau) \in E} f\left(\left(t_{\sigma}, t_{\tau}\right),\left(y_{\sigma}, y_{\tau}\right) \mid \mathbf{w}\right)}_{\text {Edge scores: RO information }},
$$

where the function $f$ outputs an edge score for each candidate assignment pair $\left(y_{\sigma}, y_{\tau}\right)$ given the observed RTs $\left(t_{\sigma}, t_{\tau}\right)$ and the derived RO (Figure $\left.1 \mathbf{d}\right)$. The edge score expresses the agreement between the observed and the predicted RO for a candidate pair, i.e. if a candidate pair receives a high score it is more likely to be correct. Function $f$ is parameterized by the vector $\mathbf{w}$, which is trained specifically for each $\mathrm{MS}^{2}$ scorer (see next section). Using the compatibility score function $F$ (Equation (1)) we compute the max-marginals 42 for each candidate and MS features. The max-marginal score of a particular candidate $m \in \mathcal{C}_{\sigma}$ and MS feature $\sigma$ is defined as the maximum compatibility score that a candidate assignment $\overline{\mathbf{y}} \in \Sigma$ with $\bar{y}_{\sigma}=m$ can reach:

$$
\mu\left(y_{\sigma}=m \mid \mathbf{x}, \mathbf{t}, \mathbf{w}, G\right)=\max _{\left\{\overline{\mathbf{y}} \in \Sigma: \bar{y}_{\sigma}=m\right\}} F(\overline{\mathbf{y}} \mid \mathbf{x}, \mathbf{t}, \mathbf{w}, G) .
$$

We use $\mu$ to rank the molecular candidates [34]. For general graphs $G$ the max-marginal inference problem (MMAP) is intractable in practice due to the exponential size of the label space $\Sigma$. Therefore, we approximate the MMAP problem by performing the inference on tree-like graphs $T_{k}$ randomly sampled from $G$ (Figure 1 $c$ ), for which exact inference is feasible [42, 43. Subsequently, we average the max-marginal scores $\mu\left(y_{\sigma}=m \mid \mathbf{x}_{i}, \mathbf{t}_{i}, \mathbf{w}_{k}, T_{k}\right)$ over a set of trees $\mathbf{T}$, an approach that performed well for practical applications 44, 45, 34]. For each spanning tree $T_{k}$, we apply a separately trained SSVM model $\mathbf{w}_{k}$ to increase the diversity of the predictions.

Joint annotation using Structured Support Vector Machines (SSVM). We propose to tackle the joint assignment of candidate labels $\mathbf{y} \in \Sigma$ to the sequence of MS features of a LC$\mathrm{MS}^{2}$ experiment through structured prediction, a family of machine learning methods generally used to annotate sequences or networks $35,46,45]$. In our model, the structure is given by the observed RO of the MS feature pairs $\left(y_{\sigma}, y_{\tau}\right)$, which provides additional information on the correct candidate labels $y_{\sigma}$ and $y_{\tau}$. Given a set of annotated LC-MS ${ }^{2}$ experiments extracted from MassBank 37] (Figure 1e), we train a Structured Support Vector Machine (SSVM) 35 model w predicting the edge scores. SSVMs models can be optimized using the max-margin principle 35]. In a nutshell, given a set of ground truth annotated MS feature sequences, the model parameters $\mathbf{w}$ are optimized such that the correct label sequence $\mathbf{y}_{i} \in \Sigma_{i}$, that is the structure annotations for all MS features in an LC-MS ${ }^{2}$ experiment, scores higher than any other possible label sequence assignment $\mathbf{y} \in \Sigma_{i}$ (Figure 1f). 


\section{Results}

This section describes our experiments and the corresponding results with LC-MS ${ }^{2}$ Struct. We start with a description of the training and evaluation data extracted from MassBank. Then, we continue with a comparison of LC-MS ${ }^{2}$ Struct to other approaches for $\mathrm{MS}^{2}$ and RT or RO score integration. Subsequently, we go into more details by analysing the performance of LC-MS ${ }^{2}$ Struct for different molecular classes. We conclude with a study of our method applied for the ranking of candidate sets including stereoisomers.

Extracting training data from MassBank. For this study we extracted ground truth annotated $\mathrm{MS}^{2}$ spectra and RTs from MassBank 37], a public online database for $\mathrm{MS}^{2}$ data. Each individual MassBank record typically provides a rich set of meta information (see Extended Data), such as the chromatographic and MS conditions as well as molecular structure annotations. To train the SSVM model of LC-MS ${ }^{2}$ Struct, we need sets of MS features, i.e. (MS $\left.{ }^{2}, \mathrm{RT}\right)$-tuples, with ground truth structure annotations as available in MassBank. We process the MassBank data such that the experimental conditions are consistent within each MS feature set. That means, for example, that the LC setup is identical, such that we can compare the RTs within the set to derive the ROs, or that the same MS configuration was used, as we would assume in a typical LC-MS ${ }^{2}$ experiment. We developed a Python package "massbank2db" 47] that can process MassBank records and groups them into consistent MS feature sets, which we denote as MB-subsets. For the SSVM training and the evaluation of LC-MS ${ }^{2}$ Struct, as well as comparison methods, we sample sequences of MS features to simulate LC-MS ${ }^{2}$ experiments in which we measure the signal of multiple unknown compounds under consistent experimental setups. Figure 10 illustrates the grouping and LC-MS ${ }^{2}$ sampling process. Two collections of MassBank data were considered: ALLDATA and the ONLYSTEREO subset. Further details can be found in the Methods section.

Comparison of LC-MS ${ }^{2}$ Struct with other approaches. In the first set of experiments we compare LC-MS ${ }^{2}$ Struct with previous approaches for candidate ranking either using only $\mathrm{MS}^{2}$ or additionally RT or RO information: Only-MS $S^{2}$ uses the $\mathrm{MS}^{2}$ spectrum information to rank the molecular candidates and serves as baseline; $M S^{2}+R O$ 34 uses a Ranking Support Vector Machine (RankSVM) 48, 49 to predict the ROs of candidate pairs and a probabilistic inference model to combine the ROs with $\mathrm{MS}^{2}$ scores; $M S^{2}+R T$ uses predicted RTs to remove false positive molecule structures from the candidate set, ordered by their $\mathrm{MS}^{2}$ score, by comparing the predicted and observed RT; $M S^{2}+\log P$ is an approach introduced by Ruttkies et al. 11], which uses the observed RT to predict the XLogP3 value 50 of the unknown compound and compares it with the candidates' XLogP3 values extracted from PubChem to refine the initial ranking based on the $\mathrm{MS}^{2}$ scores. A detailed description of the comparison approaches can be found in the Methods section. The RO based methods (LC-MS ${ }^{2}$ Struct and $\mathrm{MS}^{2}+\mathrm{RO}$ ) were trained using the RTs from all available MB-subsets, at the same time ensuring that no test molecular structure (based on InChIKey first block) was used for the model training (structure disjoint). On the other hand, for the RT based approaches $\left(\mathrm{MS}^{2}+\mathrm{RT}\right.$ and $\mathrm{MS}^{2}+\log P$ ), the RT and XLogP3 predictors were trained in a structure disjoint fashion, using only the RT data available for that respective MB-subset. For the experiment, all MB-subsets with more than $75\left(\mathrm{MS}^{2}, \mathrm{RT}\right)$-tuples from the ALLDATA data setup were used, as the RT based approaches require target LC system-specific RT training data (see Extended Data). The ranking performance was computed for each LC-MS ${ }^{2}$ experiment within a particular MB-subset. The molecules in the candidate sets are identified by their InChIKey first block (i.e. the structural skeleton). That means, there are no stereoisomers in the candidate set and the rank of the ground truth molecular structure is determined using the InChIKey first block. Each candidate ranking approach was evaluated with three state-of-the-art $\mathrm{MS}^{2}$ scorers: CFM-ID 4.0 [18, MetFrag [11] and SIRIUS 17]. Further details can be found in the Methods section.

Figure $2 \mathrm{a}$ shows the average ranking performance (top-k accuracy) across 350 LC-MS ${ }^{2}$ experiments, with each encompassing about $50\left(\mathrm{MS}^{2}, \mathrm{RT}\right)$-tuples (see Methods). For CFM-ID and MetFrag, LC-MS ${ }^{2}$ Struct provides 3.1 and 4.5 percentage unit increases over the Only-MS ${ }^{2}$ for 
the top-1 accuracy, corresponding to $53.5 \%$ and $66.1 \%$ performance gain. In our setting, that translates to 1.6 respectively 2.3 additional identifications at the top rank (out of approx. 50). The performance improvement increases for larger $k$, reaching as far as 7.2 and 8.6 percentage units at top-20, which means 3.6 respectively 4.3 additional correct structures in the top-20. For SIRIUS, the improvements are only modest, on average around 0.5 percentage units for top- 1 to top-20. The runner-up score integration method is $\mathrm{MS}^{2}+\mathrm{RO}$, which also makes use of predicted ROs. Combined with SIRIUS, $\mathrm{MS}^{2}+\mathrm{RO}$ actually achieves the best molecule ranking performance of all considered methods. For CFM-ID and MetFrag it leads to about half of the performance gain as LC-MS ${ }^{2}$ Struct. The approaches relying on RTs, either by candidate filtering $\left(\mathrm{MS}^{2}+\mathrm{RT}\right)$ or through $\log P$ prediction $\left(\mathrm{MS}^{2}+\log P\right.$ ), only lead to a tiny improvement for MetFrag and CFMID, but none for SIRIUS, for which we even observe $\mathrm{MS}^{2}+\mathrm{RT}$ leading to a decrease in ranking performance by about 2 percentage units. An explanation for this is that the filtering approach removes on average $4.7 \%$ of the correct candidates, which leads to false negative predictions.

The performance gain by using either RO or RT varies between the MB-subsets that differ by their LC-MS ${ }^{2}$ setup (see Supplementary Table 4) and compound class composition (see Extended Data). We illustrate these differences in Figure $2 \mathbf{b}$. Applying LC-MS ${ }^{2}$ Struct improves the ranking performance in almost all MB-subsets, including the SIRIUS data (some very slight decreases were observed in some SIRIUS sets). This is in stark contrast to the RT based approaches $\left(\mathrm{MS}^{2}+\mathrm{RT}\right.$ and $\mathrm{MS}^{2}+\log P$ ), which often lead to less accurate rankings, especially for SIRIUS. Furthermore, as can be seen already from the average results (Figure 2a), the benefit of LC-MS ${ }^{2}$ Struct depends on the $\mathrm{MS}^{2}$ base scorer. For example, the top-1 accuracy of the subsets "AC_003" and "NA_003" can be greatly improved for MetFrag but show little or no improvement for CFM-ID. Interestingly, both datasets are natural product toxins, which are perhaps poorly explained by the bond-disconnection approach of MetFrag (often observed for substances with many rearrangements). On the other hand, for "RP_001" and "LQB_000" the largest improvements can be reached for CFM-ID. The RT filtering approach ( $\mathrm{MS}^{2}+\mathrm{RT}$ ) performs particularly well for "LQB_000" and "UT_000". These subsets are characterized by a relatively homogeneous set of molecules in terms of ClassyFire [51] super-classes (see Extended Data), encompassing mostly lipids and lipid-like molecules. Since the RT prediction models are trained using only data from the respective MB-subset, this can lead to more accurate models for subsets with less heterogeneous sets of molecules. Hence, the RT filtering could work well in such cases 26 .

Performance analysis of LC-MS ${ }^{2}$ Struct for different compound classifications. Our next experiment investigates how LC-MS ${ }^{2}$ Struct can improve the identification across different categories in two molecule classification systems. The first system is the ClassyFire [51 taxonomy, which we use to assign molecule classes to all ground truth structures in our evaluation set. As a second classification system, we use the one provided by PubChemLite [39. Figure 3 shows the average top- 1 and top- 20 accuracy improvement of LC-MS ${ }^{2}$ Struct over the Only-MS ${ }^{2}$ baseline for each ClassyFire super-class and PubChemLite annotation category (see Methods). For ClassyFire (Figure 3a), we observe that the ranking performance improvement for the different super-classes depends on the $\mathrm{MS}^{2}$ scorer. For example, the top- 1 accuracy of "Alkaloids and derivatives" can be improved by 6.7 percentage units for MetFrag, but improves only very little for CFM-ID and SIRIUS (about 1 percentage unit). The picture looks different for "Organic oxygen compounds", for which the top- 1 accuracy improves by about 4.7 percentage units when using CFM-ID, but little to no improvement is observed for the other $\mathrm{MS}^{2}$ scorers. This suggests that the CFM-ID results may be improved with the inclusion of more "Organic oxygen compounds". On the other hand, it seems that the "Alkaloids and derivatives", "Organic acids and derivatives" and "Organic nitrogen compounds" may be less well explained by MetFrag (perhaps with more rearrangements, or less distinguishable spectra), such that the improvement from the RO approach is more apparent.

For the PubChemLite classification (Figure 3b) we also see that different $\mathrm{MS}^{2}$ scorers benefit differently by using LC-MS ${ }^{2}$ Struct. The improvement seems generally more consistent across the annotation categories, with one or two differing exceptions for MetFrag and CFM-ID. The SIRIUS performance seems unaffected, irrespective of the annotation category. Looking at the top-1 
cases: For CFM-ID, the biggest improvement is in the "Food Related" category. For MetFrag, the category that improved the most with LC-MS²Struct was "Agrochemicals", whereas both "Agrochemicals" and "Identification" showed the least improvement for CFM-ID. The performance was relatively consistent over the other categories. For the top-20 cases, the performance seems relatively consistent except for the "Food related" (as for top-1) and "noClassification" cases. The low performance gain achieved by LC-MS ${ }^{2}$ Struct for molecules not covered in PubChemLite ("noClassification") could be due to the fact that one third of the "noClassification" molecules belong to the ClassyFire class "Glycerophospholipids". As shown in Extended Data Figure 6, this class does not benefit from LC-MS'Struct, unlike other lipid classes also shown in that figure.

Annotation of stereoisomers. In general, $\mathrm{MS}^{2}$ alone cannot reliably distinguish between stereoisomers 5, 24. Thus $\mathrm{MS}^{2}$ scorers mostly output the same matching score between spectrum and candidate molecule for different stereoisomers (c.f. [7, 17]). However, there is a difference between stereoisomers that vary in their double-bond orientation (e.g. cis-trans or $E$ - $Z$ isomerism), which may have different shapes and thus exhibit different fragmentation and/or interactions with the LC system in some cases (see Figure $5 \mathrm{a}$ ), compared with stereoisomers involving chiral centres (e.g. $R, S$ isomers), which may not exhibit such dramatic differences in regular $\mathrm{LC}^{-\mathrm{MS}^{2}}$ experiments. Thus, in our last experiment we study whether LC-MS ${ }^{2}$ Struct can annotate stereoisomers more accurately than $\mathrm{MS}^{2}$ alone. For that we consider candidate sets containing stereoisomers and evaluate LC-MS ${ }^{2}$ Struct only using MassBank records where the ground truth structure has stereochemistry information provided, i.e. where the InChIKey second block is not "UHFFFAOYSA" (the ONLYSTEREO data setup, see Methods). The molecular candidates are represented using two different molecular fingerprint features: One that includes stereochemistry information (3D); and one that omits it (2D) (see Methods). This allows us to assess the importance of the stereochemistry encoding of features for the candidate ranking.

Figure $4 \mathrm{a}$ shows the ranking performance of $\mathrm{LC}-\mathrm{MS}^{2}$ Struct, using $2 \mathrm{D}$ respectively $3 \mathrm{D}$ fingerprints, compared with the Only-MS ${ }^{2}$ baseline. It can be seen that LC-MS ${ }^{2}$ Struct improves the ranking for all three $\mathrm{MS}^{2}$ scorers. The improvement, however, is notably larger when using candidate features that encode stereochemistry (3D). That demonstrates that LC-MS ${ }^{2}$ Struct can use the RO information to improve the annotation of stereoisomers, but that the molecular features need to encode stereochemistry to achieve the best performance. When looking into the top- 1 performance of LC-MS ${ }^{2}$ Struct (3D) for the individual $\mathrm{MS}^{2}$ scorers, we observe an improvement by 2.6, 3.8 and 3.2 percentage units for CFM-ID, MetFrag and SIRIUS, respectively. This translates to performance gains of $87.3 \%, 95.9 \%$ and $44.3 \%$ with about 1.5 additional structures correctly ranked at top rank (1) for all three $\mathrm{MS}^{2}$ scorers. In contrast to our previous experiments, we see that LC-MS ${ }^{2}$ Struct can also improve the ranking when SIRIUS is used as MS $^{2}$ scorer.

\section{Discussion}

We have presented LC-MS ${ }^{2}$ Struct, a novel approach for the integration of tandem mass spectrometric and liquid chromatography data for the structural annotation of small molecules. The method learns from the pairwise dependencies in the retention order of MS features within similar LC configurations and can generalize across different, heterogeneous LC configurations. The annotation accuracies are far superior to more traditional retention time (RT) filtering and $\log P$-based approaches, and also markedly better than previous methods that rely on retention orders. In particular, compared to Bach et al. 34, who used a graphical model as a post-hoc integration tool of $\mathrm{MS}^{2}$ scores and retention order predictions, the benefits of learning the parameters of the graphical model are clear. We note that it would in principle be possible to also train the $\mathrm{MS}^{2}$ score part (the node scores) of the model, instead of relying on separate $\mathrm{MS}^{2}$ scorers such as SIRIUS, MetFrag and CFM-ID. Such an approach could potentially further improve the results by learning from dependencies between $\mathrm{MS}^{2}$ and RO features. However, as the $\mathrm{MS}^{2}$ scorers used here are already relatively mature and well-known in the community, we have left this research line open for future efforts. 
Most $\mathrm{MS}^{2}$ scorers neglect stereochemistry, or collapse their results into one result for all stereoisomers by InChIKey first block. In our experiments, we could demonstrate that LC$\mathrm{MS}^{2}$ Struct can improve the identification of stereoisomers. The top- 1 accuracy increased by 2.6 to 3.8 and the top-20 by even 4.6 to 9.2 percentage units. Furthermore, we demonstrated that the encoding of stereochemical features in the molecule representation is essential to improved the identification of stereoisomers. These can be split into two general cases: those features encoding double-bond stereochemistry (SMILES: "Y" and "/") as well as the chiral centre configuration (SMILES: "®" and "®@"). Inspecting individual examples revealed that LC-MS ${ }^{2}$ Struct can separate the former cases with varying double-bond stereochemistry - i.e. E/Z- and cis/trans-isomers (see e.g. Figure 5). However, we note that there were very few examples of double-bond and/or chiral isomers measured on the same LC system in our dataset, which makes it difficult to verify these initial results, or interrogate these further - until such data is publicly available. Certain stereoisomers differing only in chiral centres (i.e. containing "๑" and "@@") can generally only be separated using chiral column chromatography. MassBank, and hence our datasets, currently does not cover such columns. Since MassBank also contains many metabolomics (biological) datasets with primarily naturally-observed chiral forms, some of the observed improvement could also be related to biases in our dataset. In other words, certain chiral configurations might be overrepresented in public databases (i.e. in this case MassBank), hence these are more likely to be predicted. Overall, these results suggest that LC-MS ${ }^{2}$ annotation may be improved by the use of stereochemistry information, but that a selective fingerprint definition capturing only the stereochemistry that is relevant for non-chiral LC systems should be used or developed to investigate this further.

We developed a processing pipeline to extract ground truth annotated $\mathrm{MS}^{2}$ spectra with RT information from MassBank. The $\left(\mathrm{MS}^{2}, \mathrm{RT}\right)$-tuples are grouped into subsets with homogeneous MS- and LC-conditions. This enables researchers to use MassBank data in a format suitable for machine learning, and hence can facilitate the develop of novel approaches integrating $\mathrm{MS}^{2}$ and RT information for structure annotation. We made the pipeline available to the research community in a separate Python package "massbank2db" 47.

\section{Methods}

Notation. We use the following notation to describe LC-MS² Struct:

Sequence of spectra

Sequence of retention times

Sequence of candidate sets

Sequence of labels

Candidate assignment space

$$
\begin{aligned}
& \mathbf{x}=\left(x_{1}, \ldots, x_{L}\right) \\
& \mathbf{t}=\left(t_{1}, \ldots, t_{L}\right) \\
& \mathcal{C}=\left(\mathcal{C}_{1}, \ldots, \mathcal{C}_{L}\right) \\
& \mathbf{y}=\left(y_{1}, \ldots, y_{L}\right) \in \Sigma \\
& \Sigma=\mathcal{C}_{1} \times \ldots \times \mathcal{C}_{L}
\end{aligned}
$$

where $\mathcal{X}$ and $\mathcal{Y}$ denote the $\mathrm{MS}^{2}$ spectra and the molecular structure space, respectively, and $\mathcal{C}$ denotes a candidate set that is a sub-set of all possible molecular structures, and $A \times B$ denotes cross product of two sets $A$ and $B$. For the purpose of model training and evaluation, we assume a dataset with ground truth labeled MS feature sequences: $\mathcal{D}=\left\{\left(\left(\mathbf{x}_{i}, \mathbf{t}_{i}\right), \mathcal{C}_{i}, \mathbf{y}_{i}\right)\right\}_{i=1}^{N}$, where $N$ denotes the total number of sequences. We use $i, j \in \mathbb{N}_{\geq 0}$ to index MS feature sequences and $\sigma, \tau \in \mathbb{N}_{\geq 0}$ as indices for individual MS features within a sequence, e.g. $x_{i \sigma}$ denotes the $\mathrm{MS}^{2}$ spectrum at index $\sigma$ in the sequence $i$. The length of a sequence of MS features is denoted with $L$. We denote the ground truth labels (candidate assignment) of sequence $i$ with $\mathbf{y}_{i}$ and any labelling with $\mathbf{y}$. Both, $\mathbf{y}_{i}$ and $\mathbf{y}$ are in $\Sigma_{i}$. We use $y$ to denote the candidate label variable, whereas $m$ denotes a particular molecular structure. For example, $y_{\sigma}=m$ means, that we assign the molecular structure $m$ as label to the MS feature $\sigma$.

Graphical model for joint annotation of MS features. We consider the molecular annotation problem for the output of an LC-MS ${ }^{2}$, that means assigning a molecular structure to each MS feature, as a structured prediction problem [35, 46, 45], relying on a graphical model representation 
of the sets of MS features arising from an LC-MS ${ }^{2}$ experiment. For each MS feature $\sigma$ we want to predict a label $y_{\sigma}$ from a fixed and finite candidate (label) set $\mathcal{C}_{\sigma}$. We model the observed retention orders (RO) between each MS feature pair $(\sigma, \tau)$ within an LC-MS ${ }^{2}$ experiment, as pairwise dependencies of the features. We define an undirected graph $G=(V, E)$ with the vertex set $V$ containing a node $\sigma$ for each MS feature and the edge set $E$ containing an edge for each MS feature pair $E=\{(\sigma, \tau) \mid \sigma, \tau \in V, \sigma \neq \tau\}$ (c.f. Figure 1 a and $\mathbf{c}$ ). The resulting graph is complete with an edge between all pairs of nodes. This allows us to make use of arbitrary pairwise dependencies, instead of limiting to, say, adjacent retention times. This modeling choice was previously shown to be beneficial by Bach et al. [34. Here we extend that approach by learning from the pairwise dependencies to optimize joint annotation accuracy, which leads to markedly improved annotation accuracy.

For learning, we define a scoring function $F$ that, given the input MS feature sequences $(\mathbf{x}, \mathbf{t})$ and its corresponding sequence of candidate sets $\mathcal{C}$, computes a compatibility score between the measured data and any possible sequence of labels $\mathbf{y} \in \Sigma$ :

$$
F(\mathbf{y} \mid \mathbf{x}, \mathbf{t}, \mathbf{w}, G)=\frac{1}{|V|} \sum_{\sigma \in V} \theta\left(x_{\sigma}, y_{\sigma}\right)+\frac{1}{|E|} \sum_{(\sigma, \tau) \in E}\left\langle\mathbf{w}, \Gamma\left(\mathbf{t}^{\sigma \tau}, \mathbf{y}^{\sigma \tau}\right)\right\rangle,
$$

where $\theta: \mathcal{X} \times \mathcal{Y} \rightarrow(0,1]$ is a function returning an $\mathrm{MS}^{2}$ matching score between the spectrum $x_{\sigma}$ and a candidate $y_{\sigma} \in \mathcal{C}_{\sigma},\langle\cdot, \cdot\rangle$ denotes the inner product, and $\mathbf{w}$ is a model weight vector to predict the RO matching score, based on the joint feature vector $\Gamma: \mathbb{R}_{\geq 0} \times \mathbb{R}_{\geq 0} \times \mathcal{Y} \times \mathcal{Y} \rightarrow \mathcal{F}$ between the observed RO derived from $\mathbf{t}^{\sigma \tau}=\left(t_{\sigma}, t_{\tau}\right)$ and a pair of molecular candidates $\mathbf{y}^{\sigma \tau}=\left(y_{\sigma}, y_{\tau}\right)$.

Equation (2) consists of two parts: (1) A score computed over the nodes in $G$ capturing the $\mathrm{MS}^{2}$ information; and (2) a score expressing the agreement of observed and predicted RO computed over the edge set. We assume that the node scores are pre-computed by a $\mathrm{MS}^{2}$ scorer such as CFM-ID [18, MetFrag [1] or SIRIUS 17]. The node scores are normalized to $(0,1]$ within each candidate set $\mathcal{C}_{\sigma}$. The edge scores are predicted for each edge $(\sigma, \tau)$ using the model $\mathbf{w}$ and the joint-feature vector $\Gamma$ :

$$
\begin{aligned}
f\left(\mathbf{t}^{\sigma \tau}, \mathbf{y}^{\sigma \tau} \mid \mathbf{w}\right) & =\left\langle\mathbf{w}, \Gamma\left(\mathbf{t}^{\sigma \tau}, \mathbf{y}^{\sigma \tau}\right)\right\rangle \\
& =\left\langle\mathbf{w}, \operatorname{sign}\left(t_{\sigma}-t_{\tau}\right)\left(\phi\left(y_{\sigma}\right)-\phi\left(y_{\tau}\right)\right)\right\rangle \\
& =\operatorname{sign}\left(t_{\sigma}-t_{\tau}\right)\left\langle\mathbf{w}, \phi\left(y_{\sigma}\right)-\phi\left(y_{\tau}\right)\right\rangle,
\end{aligned}
$$

with $\phi: \mathcal{Y} \rightarrow \mathcal{F}_{\mathcal{Y}}$ being a function embedding a molecular structure into a feature space. The edge prediction function (3) will produce a height edge score, if the observed RO (i.e. $\operatorname{sign}\left(t_{\sigma}-t_{\tau}\right)$ ) agrees with the predicted one.

Using the compatibility score function (2) the predicted joint annotation for $(\mathbf{x}, \mathbf{t})$ corresponds to the the highest scoring label sequence $\hat{\mathbf{y}} \in \Sigma: \hat{\mathbf{y}}=\arg \max _{\overline{\mathbf{y}} \in \Sigma} F(\overline{\mathbf{y}} \mid \mathbf{x}, \mathbf{t}, \mathbf{w}, G)$. In practice, however, instead of only predicting the best label sequence, it can be useful to rank the molecular candidates $m \in \mathcal{C}_{\sigma}$ for each MS feature $\sigma$. That is because for state-of-the-art MS ${ }^{2}$ scorers, the annotation accuracy in the top-20 candidate list is typically much higher than for the highest ranked candidate (top-1). Our framework provides candidate rankings by solving the following problem for each MS feature $\sigma$ and $m \in \mathcal{C}_{\sigma}$ :

$$
\mu\left(y_{\sigma}=m \mid \mathbf{x}, \mathbf{t}, \mathbf{w}, G\right)=\max _{\left\{\overline{\mathbf{y}} \in \Sigma: \bar{y}_{\sigma}=m\right\}} F(\overline{\mathbf{y}} \mid \mathbf{x}, \mathbf{t}, \mathbf{w}, G) .
$$

Problem (4) returns a max-marginal $\mu$ score for each candidate $m$. That is, the maximum compatibility score any label sequence $\overline{\mathbf{y}} \in \Sigma$ with $\bar{y}_{\sigma}=m$ can achieve. One can interpret Equation (2) as the log-space representation of a unnormalized Markov Random Field probability distribution over $\mathbf{y}$ associated with an undirected graphical model $G$ [43].

Feasible inference using random spanning trees (RST). For general graphs $G$ the maximum a posterior (MAP) inference problem, that is finding the highest scoring label sequence $\mathbf{y}$ given an MS feature sequence, is an $\mathcal{N} \mathcal{P}$-hard problem [52, 53]. The max-marginals inference 
(MMAP), needed for the candidate ranking, is an even harder problem which is $\mathcal{N P}^{\mathrm{PP}}$ complete [53. However, efficient inference approaches have been developed. In particular, if $G$ is tree-like, we can efficiently compute the max-marginals using dynamic programming and the max-product algorithm 42, 43. Such tree-based approximations have shown to be successful in various practical applications $44,45,34$.

Here, we follow the work by Bach et al. 34 and sample a set of random spanning trees (RST) $\mathbf{T}=\left\{T_{k}\right\}_{k=1}^{K}$ from $G$, whereby $K$ denotes the size of the RST sample. Each tree $T_{k}$ has the same node set $V$ as $G$, but and an edge set $E(T) \subseteq E$, with $|E(T)|=L-1$, ensuring that $T$ is a single connected component and cycle free. We follow the sampling procedure used by Bach et al. 34]. Given the RST set $\mathbf{T}$ we compute the averaged max-marginals to rank the molecular candidates 34:

$$
\bar{\mu}\left(y_{\sigma}=m \mid \mathbf{x}, \mathbf{t}, \mathbf{w}, \mathbf{T}\right)=\frac{1}{K} \sum_{k=1}^{K}\left(\mu\left(y_{\sigma}=m \mid \mathbf{x}, \mathbf{t}, \mathbf{w}, T_{k}\right)-\max _{\overline{\mathbf{y}} \in \Sigma} F\left(\overline{\mathbf{y}} \mid \mathbf{x}, \mathbf{t}, \mathbf{w}, T_{k}\right)\right),
$$

where we subtract the maximum compatibility score from the marginal values corresponding to the individual trees to normalize the marginals before averaging [34. This normalization value can be efficiently computed given the max-marginals $\mu$. In our experiments, we train $K$ individual models $\left(\mathbf{w}_{k}\right)$ and associate them with the trees $T_{k}$ to increase the diversity.

The Structured Support Vector Machine (SSVM) model. To train the model parameters $\mathbf{w}$ (see equation (2)), we implemented a variant of the Structured Support Vector Machine (SSVM) 36, 35. Its primal optimization problem is given as [54:

$$
\begin{aligned}
\min _{\mathbf{w}, \boldsymbol{\xi}} & \frac{1}{2}\|\mathbf{w}\|^{2}+\frac{C}{N} \sum_{i=1}^{N} \xi_{i} \\
\text { st. } & F\left(\mathbf{y}_{i} \mid \mathbf{x}_{i}, \mathbf{t}_{i}, \mathbf{w}, G_{i}\right)-F\left(\mathbf{y} \mid \mathbf{x}_{i}, \mathbf{t}_{i}, \mathbf{w}, G_{i}\right) \geq \ell\left(\mathbf{y}_{i}, \mathbf{y}\right)-\xi_{i} \\
& \forall i \in\{1, \ldots, N\}, \forall \mathbf{y} \in \Sigma_{i},
\end{aligned}
$$

where $C>0$ being the regularization parameter, $\xi_{i} \geq 0$ is the slack variable for example $i$ and $\ell: \Sigma_{i} \times \Sigma_{i} \rightarrow \mathbb{R}_{\geq 0}$ being a function capturing the loss between two label sequences. The constraint set definition (st.) of problem (6) leads to a parameter vector $\mathbf{w}$ that is trained according to the max-margin principle [36, 35, [46], that is the score $F\left(\mathbf{y}_{i}\right)$ of the correct label should be greater than the score $F(\mathbf{y})$ of any other label sequence by at least the specified margin $\ell\left(\mathbf{y}_{i}, \mathbf{y}\right)$. Note that in the SSVM problem (6) a different graph $G_{i}=\left(V_{i}, E_{i}\right)$ can be associated to each training example $i$, allowing, for example, to process sequences of different length.

We solve (6) in its dual formulation and use the Frank-Wolfe algorithm 55 following the recent work by Lacoste-Julien et al. 54. In the supplementary material we derive the dual problem and demonstrate how to solve it efficiently using the Frank-Wolfe algorithm and RST approximations for $G_{i}$. Optimizing the dual problem enables us to use non-linear kernel functions $\lambda: \mathcal{Y} \times \mathcal{Y} \rightarrow \mathbb{R}_{\geq 0}$ measuring the similarity between the molecular structures associated with the label sequences.

The label loss function $\ell$ is defined as follows:

$$
\ell\left(\mathbf{y}_{i}, \mathbf{y}\right)=\frac{1}{\left|V_{i}\right|} \sum_{\sigma=1}^{L}\left(1-\lambda\left(y_{i \sigma}, y_{\sigma}\right)\right) .
$$

and satisfies $\ell(\mathbf{y}, \mathbf{y})=0$ (a required property [54]), if $\lambda$ is a normalized kernel, which holds true in our experiments (we used the MinMax kernel [56]).

Pre-processing pipeline for raw MassBank records. Extended Data Figure 8 illustrates our MassBank (MB) pre-processing pipeline implemented in the Python package "massbank2db" 47. First, the MassBank records' text files were parsed and the $\mathrm{MS}^{2}$ spectrum, ground truth annotation, RT and meta-information extracted. Records with missing $\mathrm{MS}^{2}$, RT or annotation 
were discarded. We use the MB 2020.11 release for our experiments. Subsequently, we grouped the MassBank records into subsets (denoted as MB-subsets) where the ( $\mathrm{MS}^{2}, \mathrm{RT}$ )-tuples have been measured under the same LC- and MS-conditions. Extended Data Table 3 summarizes the grouping criteria. In the next step, we used the InChIKey [57] identifier in MassBank to retrieve the SMILES 58 representation from PubChem 20 (1st of February 2021), rather than using the contributor-supplied SMILES. This ensures that we use a single SMILES source for the molecular candidates and ground truth annotations. Before inserting the records into our final database, we performed three more filtering steps: (1) we removed records for which the ground truth exact mass deviated too much from the calculated exact mass based on the precursor mass-per-charge $(\mathrm{m} / \mathrm{z})$ and adduct type (larger than 20ppm); (2) we removed subsets that contain less then 50 unique molecular structures; (3) we removed all records associated with the MassBank prefix LU that were potential isobars (see pull-request \#152 in the MassBank GitHub repository, https: //github.com/MassBank/MassBank-data/pull/152). Supplementary Table 4 summarizes the meta-information for all generated MB-subsets.

Generating the molecular candidate sets. We used SIRIUS [8, 17 to generate the molecular candidate sets. For each MassBank record the ground truth molecular formula was used by SIRIUS to collect the candidate structures from PubChem 20. The candidate sets generated by SIRIUS contain a single stereoisomer per candidate, identified by their InChIKey first block (structural skeleton). To study the ability of LC-MS ${ }^{2}$ Struct to annotate the stereochemical variant of the molecules, we enriched the SIRIUS candidates sets with stereoisomers. For that, the InChIKey first block of each candidate was used to search PubChem (1st of Feburary 2021) for stereoisomers. The additional molecules were then added to the candidate sets.

Pre-computing the $\mathbf{M S}^{2}$ matching scores. For each MB-subset, $\mathrm{MS}^{2}$ spectra with identical adduct type (e.g. $[\mathrm{M}+\mathrm{H}]+)$ and ground truth molecular structure were aggregated. Depending on the $\mathrm{MS}^{2}$ scorer we either merged the $\mathrm{MS}^{2}$ into a single spectrum (CFM-ID and MetFrag) following the strategy by Ruttkies et al. 11] or we provided the $\mathrm{MS}^{2}$ spectra separately (SIRIUS). To compute the CFM-ID (v4.0.7) $\mathrm{MS}^{2}$ matching score we first predicted the in silico $\mathrm{MS}^{2}$ spectra for all molecular candidate structures based on their isomeric SMILES representation using the pre-trained CFM-ID models (Metlin 2019 MSML) by Wang et al. 18. We merged the three in silico spectra predicted by CFM-ID for different collision energies and compared them with the merged MassBank spectrum using the modified cosine similarity 59 implemented in the matchms 60 (v0.9.2) Python library. For MetFrag (v2.4.5) the $\mathrm{MS}^{2}$ matching scores were calculated using the FragmenterScore feature based on the isomeric SMILES representation of the candidates. For SIRIUS, the required fragmentation trees are computed using the ground truth molecular formula of each MassBank spectrum. SIRIUS uses canonical SMILES and hence does not encode stereochemical information (canonical SMILES). Therefore, we used the same SIRIUS MS ${ }^{2}$ matching score for all stereoisomers sharing the same InChIKey first block. For all three $\mathrm{MS}^{2}$ scorers we normalized the $\mathrm{MS}^{2}$ matching scores to the range $[0,1]$ separately for each candidate set. For the machine learning based scorers (CFM-ID and SIRIUS) we predicted the matching scores such that the associated MassBank record's ground truth structures was not used for the $\mathrm{MS}^{2}$ scorer model training. If a $\mathrm{MS}^{2}$ scorer failed on a MassBank record, we assigned a constant $\mathrm{MS}^{2}$ score to each candidate.

Molecular feature representations. For LC-MS'2 Struct, we used extended connectivity fingerprints with function-classes (FCFP) 61] to represent molecular structures in our experiments. We employed RDKit (v2021.03.1) for the FCFP fingerprint generation. The fingerprints were computed based on the isomeric SMILES. RDKit parameter "useChirality" was used to generate fingerprints that either encode stereochemistry (3D) or not (2D). We used counting FCFP fingerprints. To define the set of substructures in the fingerprint vector, we first generated all possible substructures, using a FCFP radius of two, based on a set of 50000 randomly sampled molecular candidates associated with our training data, and all the ground truth training struc- 
tures, resulting in 6925 (3D) and 6236 (2D) substructures. We used 2D FCFP fingerprints in our experiments, except for the experiments focusing on the identification of stereoisomers, where we used 3D fingerprints. We used the MinMax-kernel [56] to compute the similarity between the molecules.

Computing molecular categories. For the analysis of the ranking performance for different molecular categories, we used two classification systems, ClassyFire [51], which classifies molecules according to their structure and PubChemLite 39, which focuses on molecules' relevance to exposomics. For ClassyFire, we used the "classyfireR" R package to retrieve the classification for each ground truth molecular structure in our dataset. For PubChemLite classifications, we first check for each molecular structure whether it is contained in PubChemLite by matching the InChIKey first block. We considered all 10 of the provided PubChemLite classes. If a molecular structure was not found in PubChemLite we assign it to the category "noClassification".

Training and evaluation data setups. We only considered MassBank data that has been analyzed using a LC reversed phase (RP) column. We removed molecules from the data if their measured retention time (RT) was less than three times the estimated column dead-time 62], as we considered such molecules to be non-retaining.

We considered two separate data setups. The first one, denoted by ALLDATA, used all available MassBank data to train and evaluate LC-MS ${ }^{2}$ Struct. This setup was used to compare the different candidate ranking approaches as well as to investigate the performance across various molecular classes. The second setup, denoted by ONLYSTEREO, used MassBank records where the ground truth molecular structure contains stereochemical information, i.e. where the InChIKey second block is not "UHFFFAOYSA". This setup was used in the experiments regarding the ability of LC-MS ${ }^{2}$ Struct to distinguish stereochemistry. In the training, we additionally used MassBank records that appear only without stereochemical information in our candidate sets, identified by the InChIKey second block equal to "UHFFFAOYSA" in PubChem. The number of available training and evaluation $\left(\mathrm{MS}^{2}, \mathrm{RT}\right)$-tuples per MB-subset are summarized in Extended Data Table 1.

For each MB-subset we sampled a set of LC-MS ${ }^{2}$ experiments, i.e. $\left(\mathrm{MS}^{2}, \mathrm{RT}\right)$-tuple sequences, from the available evaluation data. The number of LC-MS ${ }^{2}$ experiments ( $n$ below) depended on the number of available $\left(\mathrm{MS}^{2}, \mathrm{RT}\right)$-tuples (see Extended Data Table 1) as follows

$$
n= \begin{cases}0 & \text { if }|\mathcal{D}|<30 \\ 1 & \text { if }|\mathcal{D}| \leq 75 \\ 15 & \text { if }|\mathcal{D}| \leq 250 \\ \left\lfloor\frac{|\mathcal{D}|}{50}\right\rfloor & \text { else. }\end{cases}
$$

where $\mathcal{D}$ is a set of $\left(\mathrm{MS}^{2}, \mathrm{RT}\right)$-tuples with ground truth annotation and molecular candidate sets associated with a MB-subset. If there are less than $30\left(\mathrm{MS}^{2}, \mathrm{RT}\right)$-tuples available, we do not generate an evaluation LC-MS ${ }^{2}$ experiment from the corresponding MB-subset. Based on this sampling scheme, we obtained 354 and 94 LC-MS ${ }^{2}$ experiments for ALLDATA and ONLYSTEREO, respectively, for our evaluation (see Extended Data Table 1).

We trained eight $(K=8)$ separate SSVM models $\mathbf{w}_{k}$ for each evaluation LC-MS ${ }^{2}$ experiment. For each SSVM model we first generated a set containing the $\left(\mathrm{MS}^{2}, \mathrm{RT}\right)$-tuples from all MBsubsets. Then, we removed all tuples whose ground truth molecular structure, determined by the InChIKey first block, was in the respective evaluation LC-MS ${ }^{2}$ experiment. Lastly, we randomly sampled LC-MS ${ }^{2}$ experiments from the training tuples, within their respective MB-subset, with a length randomly chosen from $\{4, \ldots, 32\}$ (see also Figure 1e) and an RST $T_{i k}$ assigned for each MS feature sequence $i$. In total $768 \mathrm{LC}^{-\mathrm{MS}^{2}}$ training experiments were generated for each SSVM model. To speed up the model training, we restricted the candidate set size $\left|\mathcal{C}_{i \sigma}\right|$ of each training MS feature $\sigma$ to maximum 75 candidate structures by random sub-sampling. Each SSVM model $\mathbf{w}_{k}$ was applied to the evaluation LC-MS ${ }^{2}$ experiment, associated with different RSTs $T_{k}$, and the 
averaged max-marginal scores where used for the final candidate ranking (see Equation (5) and Figure 1 $\mathrm{c}$ ).

SSVM hyper-parameter optimization. The SSVM regularization parameter $C$ was optimized for each training set separately using grid search and evaluation on a random validation set sampled from the training data's (MS ${ }^{2}, \mathrm{RT}$ )-tuples (33\%). A set of LC-MS ${ }^{2}$ experiments was generated from the validation set and used to determine the Normalized Discounted Cumulative Gain (NDCG) 63 for each $C$ value. The regularization parameter with the highest NDCG value was chosen to train the final model. We used the scikit-learn 64] (v0.24.1) Python package to compute the NDCG value, taking into account ranks up until 10 (NDCG@10) and defined the relevance for each candidate to be 1 if it is the correct one and 0 otherwise. To reduce the training time, we searched the optimal $C^{*}$ only for SSVM model $k=0$ and used $C^{*}$ for the other models with $k>0$.

Ranking performance evaluation. We computed the ranking performance (top-k accuracy) for a given LC-MS ${ }^{2}$ experiment using the tie-breaking strategy described in 8: If a ranking method assigns an identical score to a set of $n$ molecular candidates, then all accuracies at the ordinal ranks $k$ at which one of these candidates is found are increased by $\frac{1}{n}$. We computed a candidate score (i.e. Only-MS ${ }^{2}$, LC-MS ${ }^{2}$ Struct, etc.) for each molecular structure in the candidate set. In the experiments using the ALLDATA setup we collapsed the candidates by InChIKey first block, assigning the maximum candidate score for each InChIKey first block group. The top-k accuracy was computed based on the collapsed candidate sets. In the ONLYSTEREO setup, we did not collapse the candidate sets before the top- $k$ accuracy computation.

For the performance analysis of individual molecule categories, either ClassyFire [51] or PubChemLite 39] classes, we first computed the rank of the correct molecular structure for each $\left(\mathrm{MS}^{2}, \mathrm{RT}\right)$-tuple of each LC-MS ${ }^{2}$ evaluation experiment based on Only-MS ${ }^{2}$ and LC-MS ${ }^{2}$ Struct scores. Subsequently, we computed the top-k accuracy for each molecule category, associated with at least 50 unique ground truth molecular structures (based on InChIKey first block). As a ground truth structure can appear multiple times in our dataset, we generate 50 random samples, each containing only one example per unique structure, and computed the averaged top-k accuracy.

Comparison of LC-MS ${ }^{2}$ Struct with other approaches. We compared LC-MS ${ }^{2}$ Struct with three different approaches to integrate tandem mass spectrum $\left(\mathrm{MS}^{2}\right)$ and retention time (RT) information, namely RT filtering, $\log P$ prediction and retention order prediction.

For RT filtering $\left(\mathrm{MS}^{2}+\mathrm{RT}\right)$, we followed Aicheler et al. 26] who used the relative error $\epsilon=$ $\frac{\left|\hat{t}-t_{\sigma}\right|}{t_{\sigma}}$, between the predicted $(\hat{t})$ and observed $\left(t_{\sigma}\right)$ retention time. We set the filtering threshold to the 95\%-quantile of the relative RT prediction errors estimated from the RT model's training data, following 27, 29. We used scikit-learn's 64 (v0.24.1) implementation of the Support Vector Regression (SVR) 65 with radial basis function (RBF) kernel for the RT prediction. For SVR, we use the same 196 features, computed using RDKit (v2021.03.1), as Bouwmeester et al. 25.

For $\log P$ prediction $\left(\mathrm{MS}^{2}+\log P\right)$ we followed Ruttkies et al. 11 who assigned a weighted sum of an $\mathrm{MS}^{2}$ and $\log P$ score $s=\beta \cdot s_{\mathrm{MS}^{2}}(m)+(1-\beta) s_{\log P}(m)$ to each candidate $m \in$ $\mathcal{C}_{\sigma}$, and use it rank the set of molecular candidates. The $\log P$ score is given by $s_{\log P}(m)=$ $\frac{1}{\delta \sqrt{2 \pi}} \exp \left(-\frac{\left(\log P_{m}-\log P_{\sigma}\right)^{2}}{2 \delta^{2}}\right)$, where $\log P_{m}$ is the predicted XLogP3 50 extracted from PubChem 20 for candidate $m$, and $\log P_{\sigma}=a \cdot t_{\sigma}+b$ is the XLogP3 value of the unknown compound, associated with MS feature $\sigma$, predicted based on its measured RT $t_{\sigma}$. The parameters $a$ and $b$ of the linear regression model were determined using a set of RT and XLogP3 tuples associated with the LC system. As Ruttkies et al. [11], we set the $\delta=1.5$ and set $\beta$ such that it optimizes the top-1 candidate ranking accuracy, calculated from a set of 25 randomly generated training LC-MS ${ }^{2}$ experiments.

For retention order prediction $\left(\mathrm{MS}^{2}+\mathrm{RO}\right)$ we used the approach by Bach et al. [34] which relies on a Ranking Support Vector Machine (RankSVM) implementation in the Python library ROSVM 
31, 66 (v0.4.0). We used counting substructure fingerprints calculated using CDK (v2.5) 67 and the MinMax kernel 56. The $\mathrm{MS}^{2}$ matching scores and predicted ROs were used to compute max-marginal ranking scores using the framework by Bach et al. 34. We used the author's implementation in version 0.2.3 68. The hyper-parameters $\beta$ and $k$ of the model were optimized for each evaluation LC-MS ${ }^{2}$ experiment separately using the respective training data. To estimate $\beta$ we generated $25 \mathrm{LC}-\mathrm{MS}^{2}$ experiments from the training data and selected the $\beta$ that maximized the Top20AUC [34] ranking performance. The sigmoid parameter $k$ was estimated using Platt's method 69] calibrated using RankSVM's training data. We used 128 random spanning trees per evaluation LC-MS ${ }^{2}$ experiment to compute the averaged max-marginals.

For the experiments comparing the different methods we used all LC-MS ${ }^{2}$ experiments generated, except the ones from the MB-subsets "CE_001", "ET_002", "KW_000" and "RP_000" (see Extended Data Table 1). For those subsets the evaluation LC-MS ${ }^{2}$ experiment contain all available $\left(\mathrm{MS}^{2}, \mathrm{RT}\right)$-tuples, leaving no LC system specific data to train the RT $\left(\mathrm{MS}^{2}+\mathrm{RT}\right)$ or $\log P$ $\left(\mathrm{MS}^{2}+\log P\right)$ prediction models. The RT and $\log P$ prediction models are trained in a structure disjoint fashion using the RT data of the particular MB-subset associated with the evaluation LC-MS ${ }^{2}$. The RO prediction model used by $\mathrm{MS}^{2}+\mathrm{RO}$ is trained structure disjoint as well, but using the RTs of all MB-subsets.

\section{Data availability}

All data used in our experiments is available online (https://zenodo.org/record/5854661). The candidate rankings of all LC-MS ${ }^{2}$ experiments are available online: ALLDATA (https:// zenodo.org/record/6036208)) and ONLYSTEREO (https://zenodo.org/record/6037629).

\section{Code availability}

The source code developed for this study is available on GitHub: Structure Support Vector Machine (SSVM) implementation (https://github.com/aalto-ics-kepaco/msms_rt_ssvm); scripts to run the experiments (https://github.com/aalto-ics-kepaco/lcms2struct_exp); and, the library implementing the MassBank pre-processing (https://github.com/bachi55/massbank2db). The candidate fingerprints where computed by the ROSVM Python library [66] (v0.4.0, https: //github.com/bachi55/rosvm) using the RDKit (2021.03.1) in the backend. The SSVM library uses the max-marginal inference solver implemented by Bach et al. [34] (v0.2.3, https: //github.com/aalto-ics-kepaco/msms_rt_score_integration).

\section{References}

[1] Ricardo R. da Silva et al. "Illuminating the dark matter in metabolomics". In: Proceedings of the National Academy of Sciences 112.41 (2015), pp. 12549-12550. ISSN: 0027-8424. DOI: 10.1073/pnas .1516878112, eprint: https : //www . pnas . org/content/112/41/12549. full.pdf. URL: https://www.pnas.org/content/112/41/12549.

[2] Alexander A. Aksenov et al. "Global chemical analysis of biology by mass spectrometry". 1 In: Nature Reviews Chemistry 1.7 (2017), p. 0054. ISSN: 2397-3358. DOI: 10.1038/s41570017-0054. URL: https://doi.org/10.1038/s41570-017-0054.

[3] Ivana Blaženović et al. "Structure annotation of all mass spectra in untargeted metabolomics". In: Analytical chemistry 91.3 (2019), pp. 2155-2162.

[4] Ivana Blaženović et al. "Software Tools and Approaches for Compound Identification of LCMS/MS Data in Metabolomics". In: Metabolites 8.2 (2018). ISSN: 2218-1989. DOI: 10.3390/ metabo8020031. URL: https://www.mdpi.com/2218-1989/8/2/31. 
[5] Emma L. Schymanski et al. "Critical Assessment of Small Molecule Identification 2016: automated methods". In: Journal of Cheminformatics 9.1 (Mar. 2017), p. 22. ISSN: 17582946. DOI: 10.1186/s13321-017-0207-1. URL: https://doi.org/10.1186/s13321-0170207-1.

[6] Dai Hai Nguyen et al. "Recent advances and prospects of computational methods for metabolite identification: a review with emphasis on machine learning approaches". In: Briefings in Bioinformatics 20.6 (Aug. 2018), pp. 2028-2043. ISSN: 1477-4054. DOI: 10.1093/bib/ bby066. eprint: https://academic . oup. com/bib/article-pdf/20/6/2028/31789414/ bby066.pdf. URL: https://doi.org/10.1093/bib/bby066

[7] Sebastian Wolf et al. "In silico fragmentation for computer assisted identification of metabolite mass spectra". In: BMC Bioinformatics 11.1 (2010), pp. 1-12. ISSN: 1471-2105.

[8] Kai Dührkop et al. "Searching molecular structure databases with tandem mass spectra using CSI:FingerID". In: Proceedings of the National Academy of Sciences (PNAS) (2015). eprint: http://www . pnas .org/content/early/2015/09/16/1509788112.full.pdf. URL: http://www.pnas.org/content/early/2015/09/16/1509788112.abstract.

[9] Felicity Allen et al. "Competitive fragmentation modeling of ESI-MS/MS spectra for putative metabolite identification". In: Metabolomics 11.1 (2015), pp. 98-110. ISSN: 1573-3882. URL: http://dx.doi.org/10.1007/s11306-014-0676-4.

[10] Céline Brouard et al. "Fast metabolite identification with Input Output Kernel Regression". In: Bioinformatics 32.12 (2016), pp. i28-i36.

[11] Christoph Ruttkies et al. "MetFrag relaunched: incorporating strategies beyond in silico fragmentation". In: Journal of Cheminformatics 8.1 (Jan. 2016), p. 3. ISSN: 1758-2946. DOI: 10.1186/s13321-016-0115-9. URL: https://doi.org/10.1186/s13321-016-0115-9.

[12] Céline Brouard et al. "Magnitude-Preserving Ranking for Structured Outputs". In: Proceedings of the Ninth Asian Conference on Machine Learning. Ed. by Min-Ling Zhang et al. Vol. 77. Proceedings of Machine Learning Research. PMLR, 15-17 Nov 2017, pp. 407-422. URL: http://proceedings.mlr.press/v77/brouard17a.html.

[13] Dai Hai Nguyen et al. "SIMPLE: Sparse Interaction Model over Peaks of moLEcules for fast, interpretable metabolite identification from tandem mass spectra". In: Bioinformatics 34.13 (2018), pp. i323-i332. DOI: 10 .1093/bioinformatics/bty252, eprint: /oup/backfile/ content_public/journal/bioinformatics/34/13/10.1093_bioinformatics_bty252/1/ bty252.pdf. URL: http://dx.doi.org/10.1093/bioinformatics/bty252.

[14] Yuanyue $\mathrm{Li}$ et al. "Identification of metabolites from tandem mass spectra with a machine learning approach utilizing structural features". In: Bioinformatics 36.4 (Oct. 2019), pp. 1213-1218. ISSN: 1367-4803. DOI: 10 . 1093/bioinformatics/btz736. URL: https: //doi.org/10.1093/bioinformatics/btz736.

[15] Christoph Ruttkies et al. "Improving MetFrag with statistical learning of fragment annotations". In: BMC bioinformatics 20.1 (2019), p. 376.

[16] Dai Hai Nguyen et al. "ADAPTIVE: leArning DAta-dePendenT, concIse molecular VEctors for fast, accurate metabolite identification from tandem mass spectra". In: Bioinformatics 35.14 (July 2019), pp. i164-i172. ISSN: 1367-4803. DOI: 10.1093/bioinformatics/btz319. eprint: https : / / academic . oup . com / bioinformatics / article-pdf / 35 / 14 / i164 / 28913118/btz319.pdf. URL: https://doi.org/10.1093/bioinformatics/btz319.

[17] Kai Dührkop et al. "SIRIUS 4: a rapid tool for turning tandem mass spectra into metabolite structure information". In: Nat Methods (2019). Doi 10.1038/s41592-019-0344-8. DOI: 10 . 1038/s41592-019-0344-8.

[18] Fei Wang et al. "CFM-ID 4.0: More Accurate ESI-MS/MS Spectral Prediction and Compound Identification". In: Analytical Chemistry 0.0 (2021). PMID: 34403256, null. DOI: 10.1021/acs . analchem.1c01465, eprint: https : //doi .org/10.1021/acs . analchem . 1c01465. URL: https://doi.org/10.1021/acs.analchem.1c01465. 
[19] David S Wishart et al. "HMDB 4.0: the human metabolome database for 2018". In: Nucleic Acids Research 46.D1 (Nov. 2017), pp. D608-D617. ISSN: 0305-1048. DOI: 10.1093/nar/ gkx1089. eprint: https://academic.oup.com/nar/article-pdf/46/D1/D608/23162277/ gkx1089.pdf. URL: https://doi.org/10.1093/nar/gkx1089

[20] Sunghwan Kim et al. "PubChem in 2021: new data content and improved web interfaces". In: Nucleic Acids Research 49.D1 (Nov. 2020), pp. D1388-D1395. ISSN: 0305-1048. DOI: 10.1093/nar/gkaa971. eprint: https://academic.oup.com/nar/article-pdf/49/D1/ D1388/35363961/gkaa971.pdf. URL: https://doi.org/10.1093/nar/gkaa971.

[21] Jan Stanstrup et al. "PredRet: Prediction of Retention Time by Direct Mapping between Multiple Chromatographic Systems". In: Analytical Chemistry 87.18 (2015). PMID: 26289378, pp. 9421-9428. URL: http://dx.doi.org/10.1021/acs . analchem.5b02287.

[22] Dorrain Yanwen Low et al. "Data sharing in PredRet for accurate prediction of retention time: Application to plant food bioactive compounds". In: Food Chemistry 357 (2021), p. 129757. ISSN: 0308-8146. DOI: https://doi.org/10.1016/j.foodchem.2021.129757. URL: https://www.sciencedirect.com/science/article/pii/S0308814621007639.

[23] S. Fanali et al. Liquid Chromatography: Fundamentals and Instrumentation. Handbooks in Separation Science. Elsevier Science, 2013. ISBN: 9780124158672.

[24] Michael Witting et al. "Current status of retention time prediction in metabolite identification". In: Journal of Separation Science 43.9-10 (2020), pp. 1746-1754. Dor: 10.1002/ jssc . 202000060 eprint: https : / / onlinelibrary . wiley . com / doi / pdf / 10 . 1002 / jssc.202000060 URL: https://onlinelibrary.wiley.com/doi/abs/10.1002/jssc. 202000060

[25] Robbin Bouwmeester et al. "Comprehensive and empirical evaluation of machine learning algorithms for small molecule LC retention time prediction". In: Analytical chemistry 91.5 (2019), pp. 3694-3703.

[26] Fabian Aicheler et al. "Retention Time Prediction Improves Identification in Nontargeted Lipidomics Approaches". In: Analytical chemistry 87.15 (2015), pp. 7698-7704.

[27] Milinda A Samaraweera et al. "Evaluation of an Artificial Neural Network Retention Index Model for Chemical Structure Identification in Nontargeted Metabolomics". In: Analytical chemistry 90.21 (2018), pp. 12752-12760.

[28] Paolo Bonini et al. "Retip: Retention Time Prediction for Compound Annotation in Untargeted Metabolomics". In: Analytical Chemistry 0.0 (2020). PMID: 32390414, null. DOI: 10.1021/acs . analchem.9b05765, eprint: https ://doi .org/10.1021/acs . analchem . 9b05765. URL: https://doi.org/10.1021/acs.analchem.9b05765.

[29] Qiong Yang et al. "Prediction of Liquid Chromatographic Retention Time with Graph Neural Networks to Assist in Small Molecule Identification". In: Anal. Chem. (Jan. 2021). ISSN: 0003-2700. DOI: 10.1021/acs . analchem.0c04071. URL: https://doi.org/10.1021/acs . analchem.0c04071

[30] Robbin Bouwmeester et al. "Generalized Calibration Across Liquid Chromatography Setups for Generic Prediction of Small-Molecule Retention Times". In: Analytical Chemistry 92.9 (2020). PMID: 32281370, pp. 6571-6578. DOI: 10.1021/acs . analchem . 0c00233 eprint: https://doi.org/10.1021/acs . analchem.0c00233. URL: https://doi.org/10.1021/ acs.analchem.0c00233.

[31] Eric Bach et al. "Liquid-chromatography retention order prediction for metabolite identification". In: Bioinformatics 34.17 (2018), pp. i875-i883. DOI: 10.1093/bioinformatics/ bty590, eprint: /oup/backfile/content_public/journal/bioinformatics/34/17/10 . 1093_bioinformatics_bty590/2/bty590.pdf. URL: http://dx . doi .org/10 . 1093/ bioinformatics/bty590. 
[32] J Jay Liu et al. "Quantitative Structure-Retention Relationships with Non-Linear Programming for Prediction of Chromatographic Elution Order". In: International journal of molecular sciences 20.14 (2019), p. 3443.

[33] Petar Žuvela et al. "Prediction of Chromatographic Elution Order of Analytical Mixtures Based on Quantitative Structure-Retention Relationships and Multi-Objective Optimization". In: Molecules 25.13 (2020), p. 3085.

[34] Eric Bach et al. "Probabilistic framework for integration of mass spectrum and retention time information in small molecule identification". In: Bioinformatics (Nov. 2020). btaa998. ISSN: 1367-4803. DOI: 10.1093/bioinformatics/btaa998 eprint: https://academic oup. com/bioinformatics/advance-article-pdf/doi/10.1093/bioinformatics/btaa998/ 34899743/btaa998.pdf. URL: https://doi.org/10.1093/bioinformatics/btaa998.

[35] I Tsochantaridis et al. "Large margin methods for structured and interdependent output variables". In: Journal of Machine Learning Research (JMLR) 6 (2005).

[36] Ben Taskar et al. "Max-Margin Markov Networks". In: Advances in Neural Information Processing Systems 16. Ed. by S. Thrun et al. MIT Press, 2004, pp. 25-32. URL: http: //papers.nips.cc/paper/2397-max-margin-markov-networks.pdf.

[37] Hisayuki Horai et al. "MassBank: a public repository for sharing mass spectral data for life sciences". In: Journal of mass spectrometry 45.7 (2010), pp. 703-714.

[38] Harry Pence et al. "ChemSpider: An Online Chemical Information Resource". In: Journal of Chemical Education 87 (Aug. 2010). DOI: 10.1021/ed100697w

[39] Emma Louise Schymanski et al. "Empowering Large Chemical Knowledge Bases for Exposomics: Pubchemlite Meets Metfrag". In: Journal of Cheminformatics (2021). ISSN: 26935015. URL: https://doi.org/10.21203/rs.3.rs-107432/v1.

[40] Andreas Schüller et al. "SMILIB: Rapid Assembly of Combinatorial Libraries in SMILES Notation". In: QSAR \& Combinatorial Science 22.7 (2003), pp. 719-721. DOI: https:// doi.org/10.1002/qsar.200310008 eprint: https://onlinelibrary.wiley.com/doi/ pdf/10.1002/qsar.200310008, URL: https://onlinelibrary.wiley.com/doi/abs/10. 1002/qsar.200310008.

[41] Andreas Schüller et al. "SmiLib v2.0: A Java-Based Tool for Rapid Combinatorial Library Enumeration". In: QSAR \& Combinatorial Science 26.3 (2007), pp. 407-410. DOI: https: //doi.org/10.1002/qsar.200630101. eprint: https://onlinelibrary.wiley.com/doi/ pdf/10.1002/qsar.200630101. URL: https://onlinelibrary.wiley.com/doi/abs/10. 1002/qsar.200630101.

[42] Martin Wainwright et al. "Tree consistency and bounds on the performance of the maxproduct algorithm and its generalizations". In: Statistics and Computing 14.2 (Apr. 2004), pp. 143-166. ISSN: 1573-1375. DOI: 10.1023/B:STC0.0000021412.33763.d5, URL: https: //doi.org/10.1023/B:STC0.0000021412.33763.d5.

[43] David J.C. MacKay. Information theory, inference and learning algorithms. Cambridge university press, 2005.

[44] Patrick Pletscher et al. "Spanning Tree Approximations for Conditional Random Fields". In: Proceedings of the Twelth International Conference on Artificial Intelligence and Statistics. Ed. by David van Dyk et al. Vol. 5. Proceedings of Machine Learning Research. Hilton Clearwater Beach Resort, Clearwater Beach, Florida USA: PMLR, 16-18 Apr 2009, pp. 408415. URL: http://proceedings.mlr.press/v5/pletscher09a.html.

[45] Hongyu Su et al. "Multilabel classification through random graph ensembles". In: Machine Learning 99.2 (2015), pp. 231-256. ISSN: 1573-0565. DOI: 10.1007/s10994-014-5465-9. URL: https://doi.org/10.1007/s10994-014-5465-9

[46] Juho Rousu et al. "Kernel-based learning of hierarchical multilabel classification models". In: Journal of Machine Learning Research 7.Jul (2006), pp. 1601-1626. 
[47] Eric Bach. massbank2db: Build a machine learning ready SQLite database from MassBank. Version 0.9.0. Jan. 2022. URL: https://github.com/bachi55/massbank2db.

[48] André Elisseeff et al. "A kernel method for multi-labelled classification". In: Advances in neural information processing systems. 2002, pp. 681-687.

[49] Thorsten Joachims. "Optimizing Search Engines Using Clickthrough Data". In: Proceedings of the Eighth ACM SIGKDD International Conference on Knowledge Discovery and Data Mining. KDD '02. Edmonton, Alberta, Canada: ACM, 2002, pp. 133-142. ISBN: 1-58113-567X. DOI: 10.1145/775047.775067, URL: http://doi.acm.org/10.1145/775047.775067.

[50] Tiejun Cheng et al. "Computation of Octanol-Water Partition Coefficients by Guiding an Additive Model with Knowledge". In: Journal of Chemical Information and Modeling 47.6 (2007). PMID: 17985865, pp. 2140-2148. DOI: 10.1021/ci700257y, eprint: https://doi. org/10.1021/ci700257y, uRL: https://doi.org/10.1021/ci700257y.

[51] Yannick Djoumbou Feunang et al. "ClassyFire: automated chemical classification with a comprehensive, computable taxonomy". In: Journal of cheminformatics 8.1 (2016), p. 61.

[52] Thomas Gärtner et al. "On structured output training: hard cases and an efficient alternative". In: Machine Learning 76.2 (2009), pp. 227-242. ISSN: 1573-0565. DOI: $10.1007 /$ s10994-009-5129-3. URL: https://doi.org/10.1007/s10994-009-5129-3.

[53] Yexiang Xue et al. "Solving Marginal MAP Problems with NP Oracles and Parity Constraints". In: Advances in Neural Information Processing Systems. Ed. by D. Lee et al. Vol. 29. Curran Associates, Inc., 2016. URL: https://proceedings.neurips.cc/paper/ 2016/file/a532400ed62e772b9dc0b86f46e583ff-Paper.pdf.

[54] Simon Lacoste-Julien et al. "Block-coordinate Frank-Wolfe optimization for structural SVMs". In: International Conference on Machine Learning. PMLR. 2013, pp. 53-61.

[55] Marguerite Frank et al. "An algorithm for quadratic programming". In: Naval Research Logistics Quarterly 3.1-2 (1956), pp. 95-110. DOI: 10.1002/nav.3800030109 eprint: https:

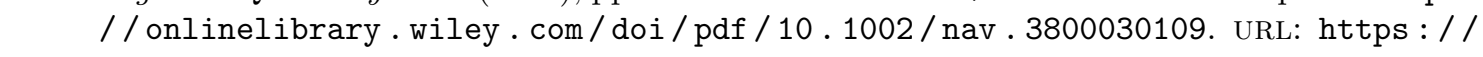
onlinelibrary.wiley.com/doi/abs/10.1002/nav.3800030109.

[56] Liva Ralaivola et al. "Graph kernels for chemical informatics". In: Neural networks 18.8 (2005), pp. 1093-1110.

[57] Stephen R. Heller et al. "InChI, the IUPAC International Chemical Identifier". In: Journal of Cheminformatics 7.1 (2015), p. 23. ISSN: 1758-2946. DOI: 10.1186/s13321-015-0068-4. URL: https://doi.org/10.1186/s13321-015-0068-4

[58] David Weininger. "SMILES, a chemical language and information system. 1. Introduction to methodology and encoding rules". In: Journal of Chemical Information and Computer Sciences 28.1 (1988), pp. 31-36. DOI: 10.1021/ci00057a005, eprint: https://pubs . acs . org/doi/pdf/10.1021/ci00057a005. URL: https://pubs .acs.org/doi/abs/10.1021/ ci00057a005.

[59] Jeramie Watrous et al. "Mass spectral molecular networking of living microbial colonies". In: Proceedings of the National Academy of Sciences 109.26 (2012), E1743-E1752. ISSN: 00278424. DOI: 10.1073/pnas.1203689109. eprint: https://www.pnas.org/content/109/26/ E1743.full.pdf. URL: https://www.pnas.org/content/109/26/E1743.

[60] Florian Huber et al. "matchms - processing and similarity evaluation of mass spectrometry data." In: Journal of Open Source Software 5.52 (2020), p. 2411. DOI: 10.21105/joss. 02411. URL: https://doi.org/10.21105/joss.02411.

[61] David Rogers et al. "Extended-Connectivity Fingerprints". In: Journal of Chemical Information and Modeling 50.5 (2010). PMID: 20426451, pp. 742-754. DOI: 10.1021/ci100050t. eprint: http://dx.doi.org/10.1021/ci100050t. URL: http://dx.doi.org/10.1021/ ci100050t. 
[62] John W. Dolan. Column Dead Time as a Diagnostic Tool. Tech. rep. 1. Jan. 2014, pp. 24-29. URL: http://www . chromatographyonline.com/column-dead-time-diagnostic-tool.

[63] Kalervo Järvelin et al. "Cumulated Gain-Based Evaluation of IR Techniques". In: ACM Trans. Inf. Syst. 20.4 (Oct. 2002), pp. 422-446. ISSN: 1046-8188. DOI: 10.1145/582415. 582418. URL: https://doi.org/10.1145/582415.582418.

[64] F. Pedregosa et al. "Scikit-learn: Machine Learning in Python". In: Journal of Machine Learning Research 12 (2011), pp. 2825-2830.

[65] Harris Drucker et al. "Support vector regression machines". In: Advances in neural information processing systems. 1997, pp. 155-161.

[66] Eric Bach. Retention Order Support Vector Machine (ROSVM). Version 0.4.0. Nov. 2021. URL: https://github.com/bachi55/rosvm.

[67] Egon L. Willighagen et al. "The Chemistry Development Kit (CDK) v2.0: atom typing, depiction, molecular formulas, and substructure searching". In: Journal of Cheminformatics

9.1 (June 2017), p. 33. ISSN: 1758-2946. DOI: 10.1186/s13321-017-0220-4. URL: https: //doi.org/10.1186/s13321-017-0220-4.

[68] Eric Bach. msmsrt_scorer: Probabilistic framework for integration of mass spectrum and retention order information. Version 0.2.3. Nov. 2021. URL: https://github.com/aaltoics-kepaco/msms_rt_score_integration

[69] John Platt. "Probabilistic outputs for support vector machines and comparisons to regularized likelihood methods". In: Advances in large margin classifiers 10.3 (June 2000).

\section{Acknowledgments}

The work by E.B. and J.R. was in part supported by Academy of Finland grants 310107 (MACOME) and 334790 (MAGITICS). E.L.S. acknowledges funding support from the Luxembourg National Research Fund (FNR) for project A18/BM/12341006 and discussions with Drs. Greg Landrum (ETHZ) and Evan Bolton (NCBI/NLM/NIH). The authors acknowledge CSC - IT Center for Science, Finland and Aalto Science-IT infrastructure, Finland for generous computational resources. E.B. thanks Dr. K. Dührkop for generating the SIRIUS candidate sets and predicting the SIRIUS MS ${ }^{2}$ scores.

\section{Authors contributions}

E.B. and J.R. designed the research. E.B. implemented the MassBank pre-processing. E.B. developed, implemented and evaluated the computational method. E.B., E.L.S. and J.R. interpreted the results. E.B., E.L.S. and J.R. wrote the manuscript.

\section{Competing interests}

The authors declare no competing interests.

\section{Additional information}




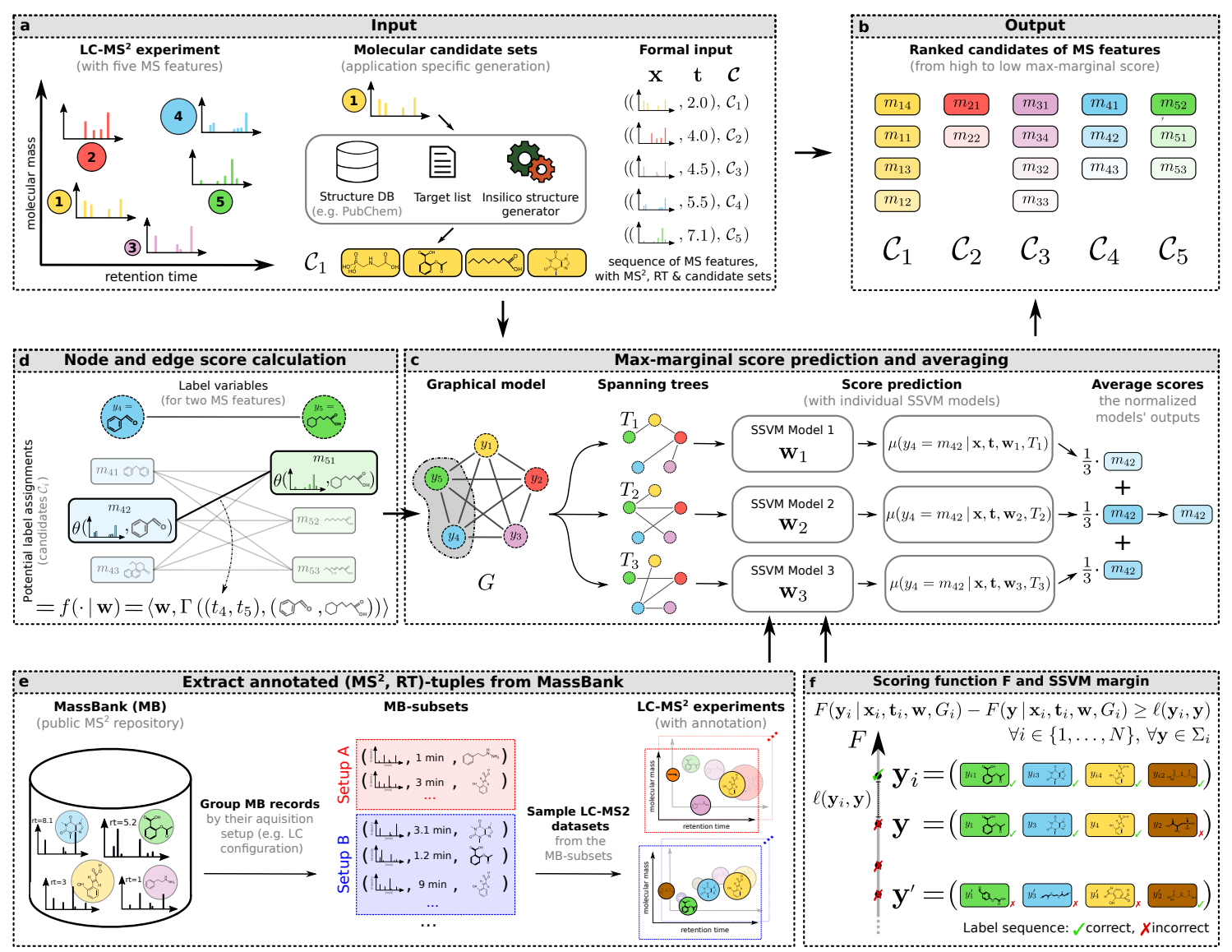

Figure 1: Overview of the LC-MS ${ }^{2}$ Struct workflow. a: Input to LC-MS ${ }^{2}$ Struct during the application phase. The LC-MS ${ }^{2}$ experiment results in a set of $\left(\mathrm{MS}^{2}, \mathrm{RT}\right)$-tuples. The MS information is used to generate a molecular candidate set for each MS feature. b: Output of LC$\mathrm{MS}^{2}$ Struct are the ranked molecular candidates for each MS feature. c: A fully connected graph $G$ models the pairwise dependency between the MS features. Using a set of random spanning trees $T_{k}$ and Structured Support Vector Machines (SSVM) we predict the max-marginal scores for each candidate used for the ranking. d: The $\mathrm{MS}^{2}$ and $\mathrm{RO}$ information is used to scores the nodes and edges in the graph $G$. e: To train the SSVM models and evaluate LC-MS ${ }^{2}$ Struct, we extract $\mathrm{MS}^{2}$ spectra and RTs from MassBank. We group the MassBank records such that their experimental setups are matching and simulate LC-MS ${ }^{2}$ experiment. f: Main objective optimized during the training of the SSVM. 
bioRxiv preprint doi: https://doi.org/10.1101/2022.02.11.480137; this version posted February 14,2022 . The copyright holder for this preprint (which was not certified by peer review) is the author/funder, who has granted bioRxiv a license to display the preprint in perpetuity. It is made available under aCC-BY-NC-ND 4.0 International license.

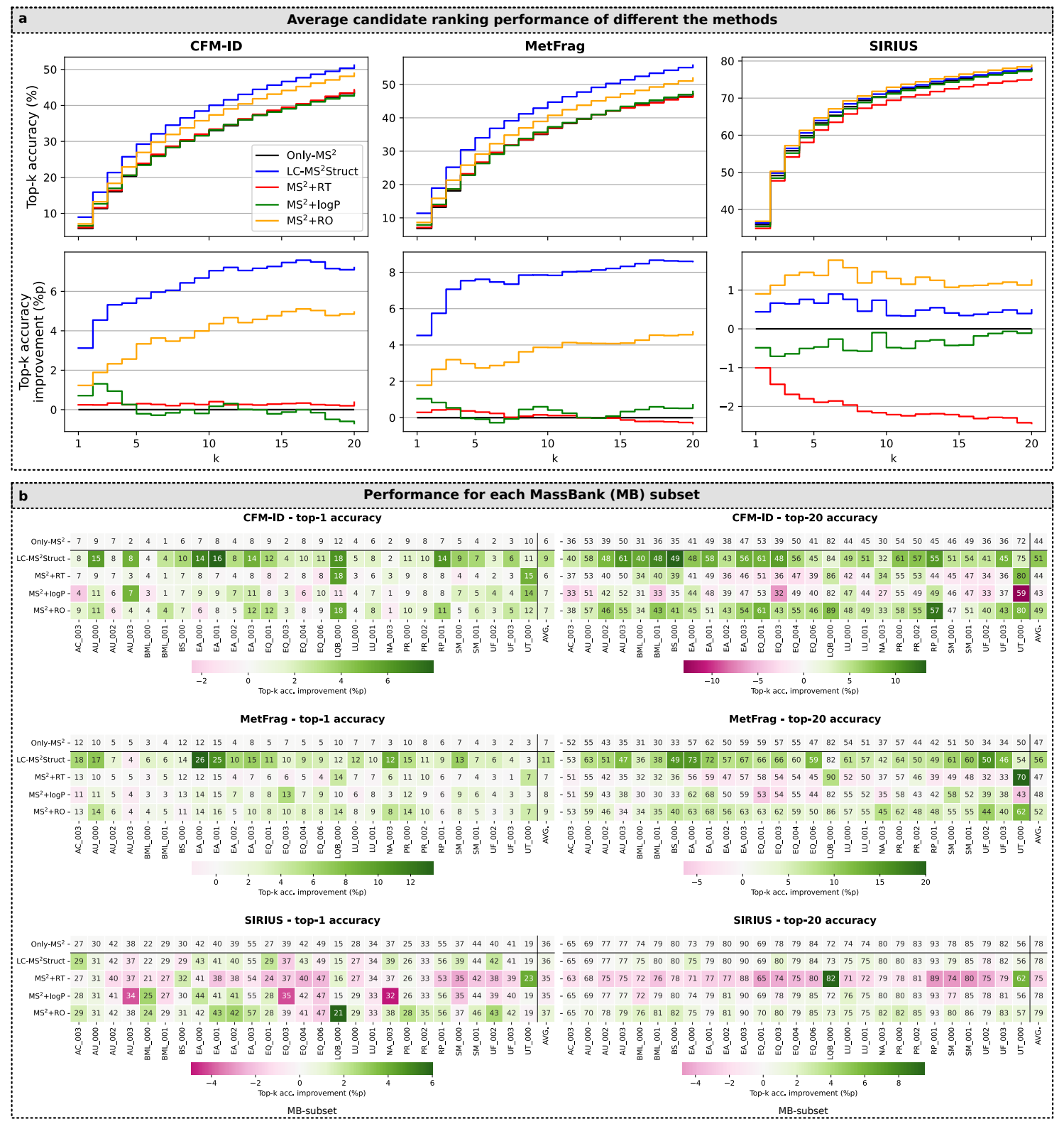

Figure 2: Different approaches to combine $\mathrm{MS}^{2}$ and retention time (RT) information: a: Comparison of the performance, measured by top-k accuracy, for the different ranking approaches combining $\mathrm{MS}^{2}$ and RT information. The results shown are averaged accuracies over 350 sample MS feature sequences (LC-MS ${ }^{2}$ experiments). b: Average top-k accuracies per MassBank (MB) subset rounded to full integers. The color encodes the performance improvement of each score integration method compared to Only-MS ${ }^{2}$. 


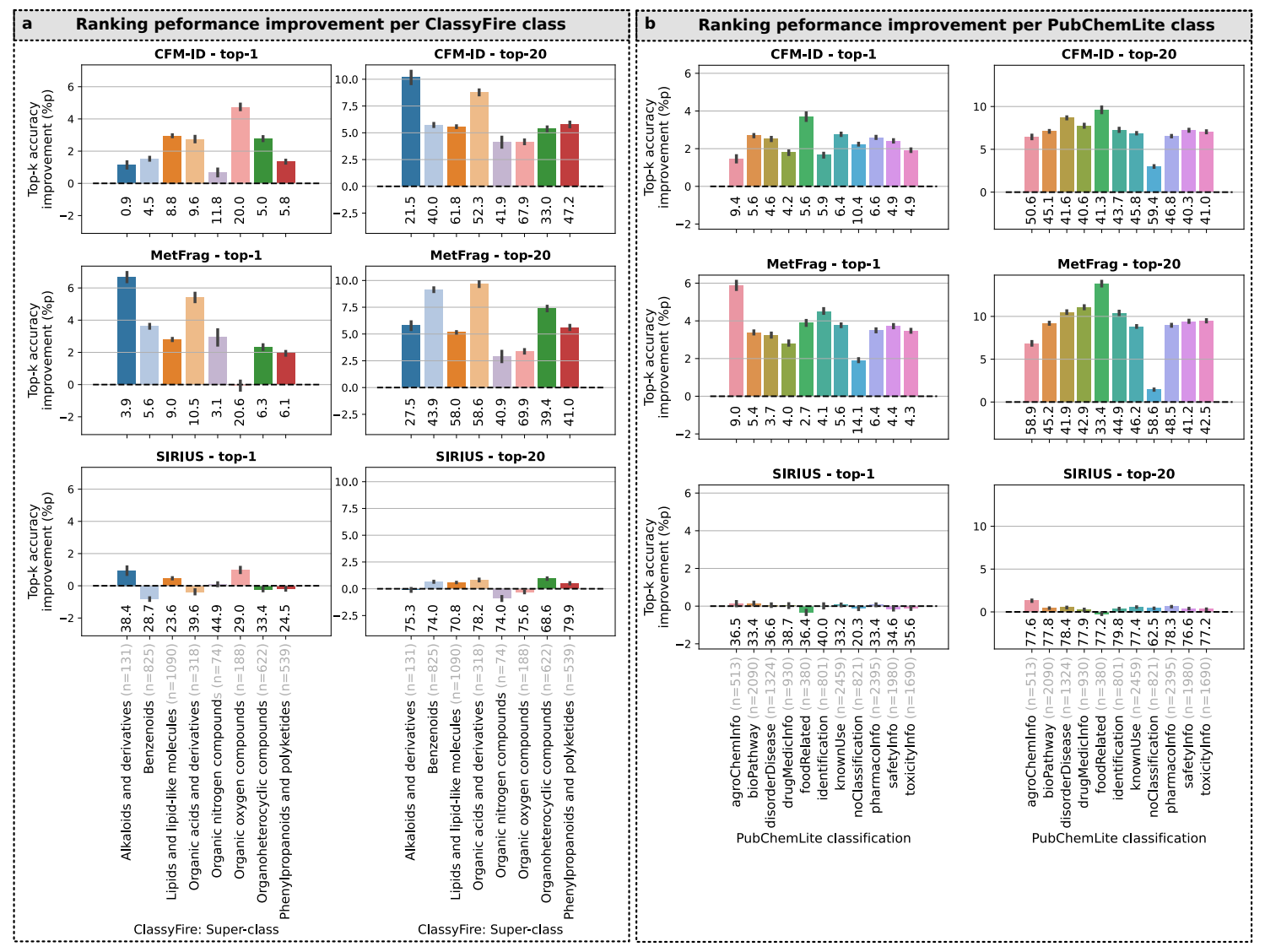

Figure 3: Performance gain by LC-MS ${ }^{2}$ Struct across molecular classes. The figure shows the average and 95\%-confidence interval of the ranking performance (top-k) improvement of LC$\mathrm{MS}^{2}$ Struct compared to Only-MS ${ }^{2}$ (baseline). The top-k accuracies (\%) under the bars show the Only-MS ${ }^{2}$ performance. For each molecular class, the number of unique molecular structures in the class is denoted in the x-axis label (n). a: Molecular classification using the ClassyFire 51 framework. b: PubChemLite [39] annotation classification system. Molecules not present in PubChemLite are summarized under the "noClassification" category. Note that in PubChemLite a molecule can belong to multiple categories. 

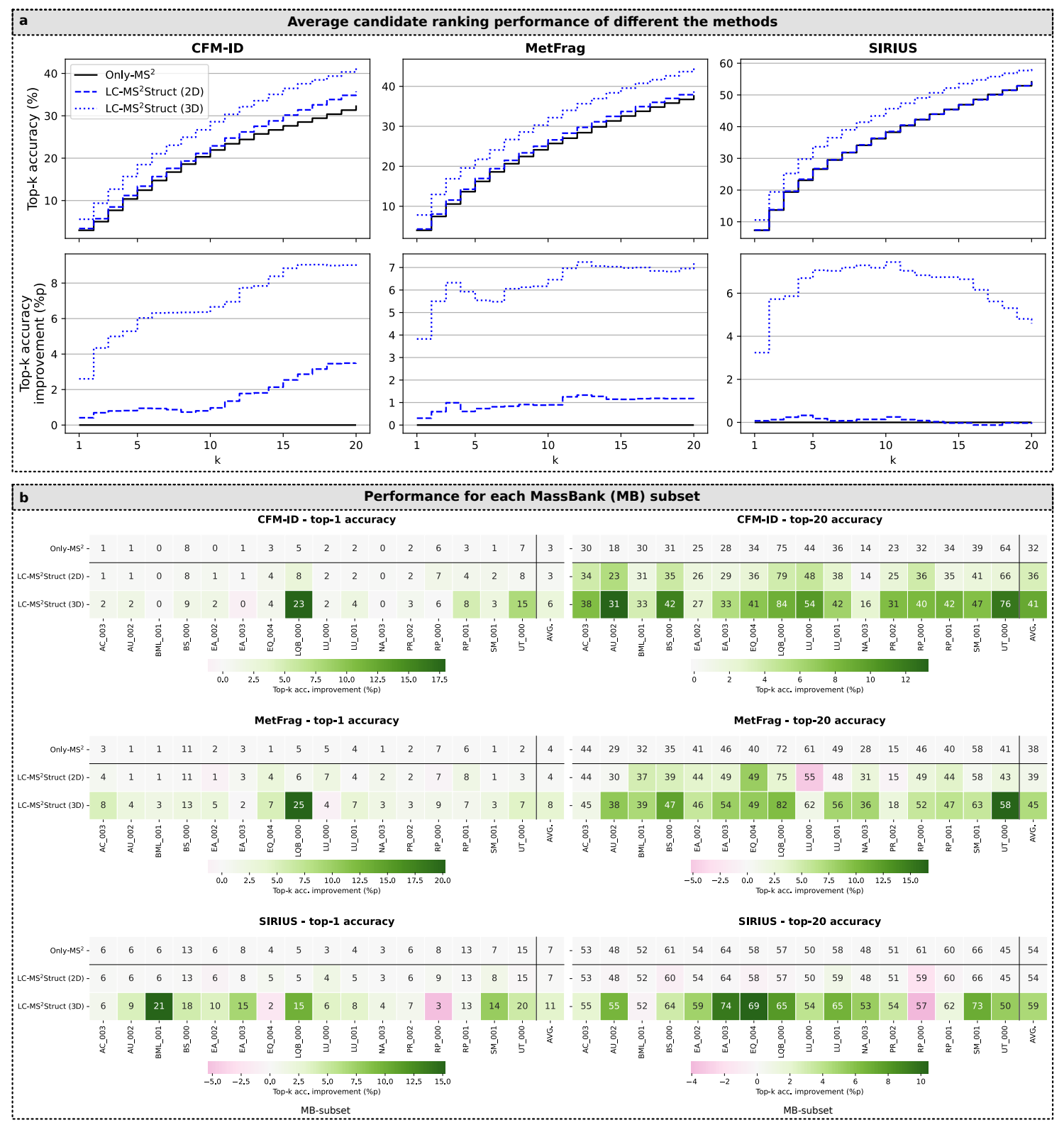

Figure 4: Using LC-MS ${ }^{2}$ Struct with different feature representations. a: Comparison of the performance, measured by top-k accuracy, of LC-MS'2 Struct using either 2D (no stereochemistry) or 3D (with stereochemistry) molecular fingerprints. The results shown are averaged accuracies over 94 sample MS feature sequences (LC-MS ${ }^{2}$ experiments). b: Average top-k accuracies per MassBank (MB) subset rounded to full integers. The color encodes the performance improvement of each score integration method compared to Only-MS ${ }^{2}$. 


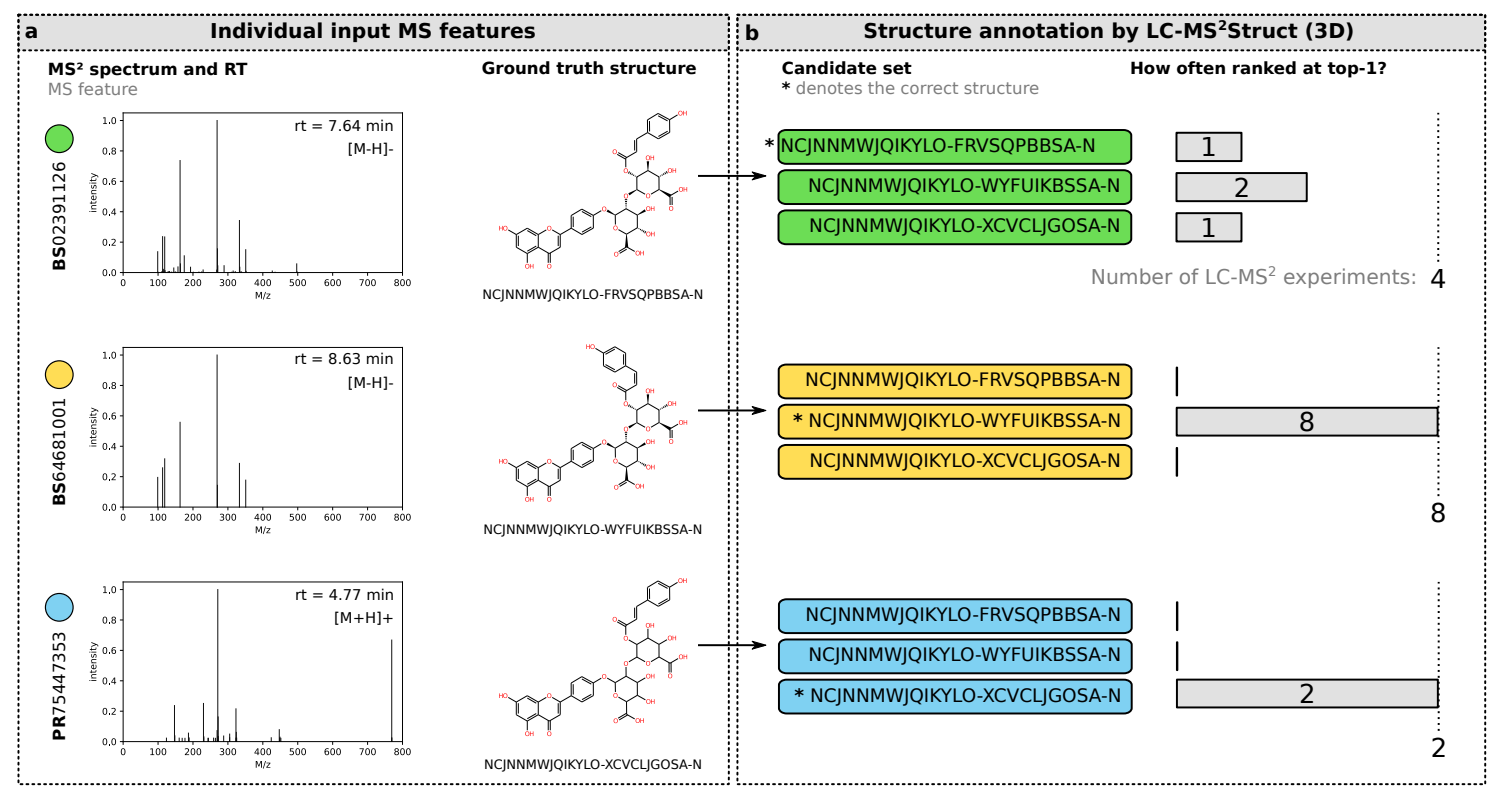

Figure 5: Application of LC-MS ${ }^{2}$ Struct to annotate stereoisomers. Post-hoc analysis of the stereoisomer annotation using LC-MS ${ }^{2}$ Struct for three $\left(\mathrm{MS}^{2}, \mathrm{RT}\right)$-tuples from our MassBank data associated with the same 2D skeleton (InChIKey first block). In our evaluation, all three MS features were analysed multiple times in different contexts (BS02391126 in 4, BS64681001 in 8 and PR75447353 in 2 LC-MS ${ }^{2}$ experiments). a: MS features with their ground truth annotations. Two of the spectra (starting with BS) were measured under the same LC condition (MB-subset "BS_000"), demonstrating the separation of E/Z-isomers on LC columns. b: The candidate sets of the three features are identical (defined by the molecular formula $\mathrm{C}_{36} \mathrm{H}_{32} \mathrm{O}_{19}$ ) and only contain three structures. For 12 out of the 14 LC-MS ${ }^{2}$ experiments, LC-MS ${ }^{2}$ Struct predicts the correct E/Z-isomer. 
bioRxiv preprint doi: https://doi.org/10.1101/2022.02.11.480137; this version posted February 14,2022 . The copyright holder for this preprint (which was not certified by peer review) is the author/funder, who has granted bioRxiv a license to display the preprint in perpetuity. It is made available under aCC-BY-NC-ND 4.0 International license.

\section{${ }_{822}$ Extended data figures and tables}


bioRxiv preprint doi: https://doi.org/10.1101/2022.02.11.480137; this version posted February 14,2022 . The copyright holder for this preprint (which was not certified by peer review) is the author/funder, who has granted bioRxiv a license to display the preprint in perpetuity. It is made available under aCC-BY-NC-ND 4.0 International license.
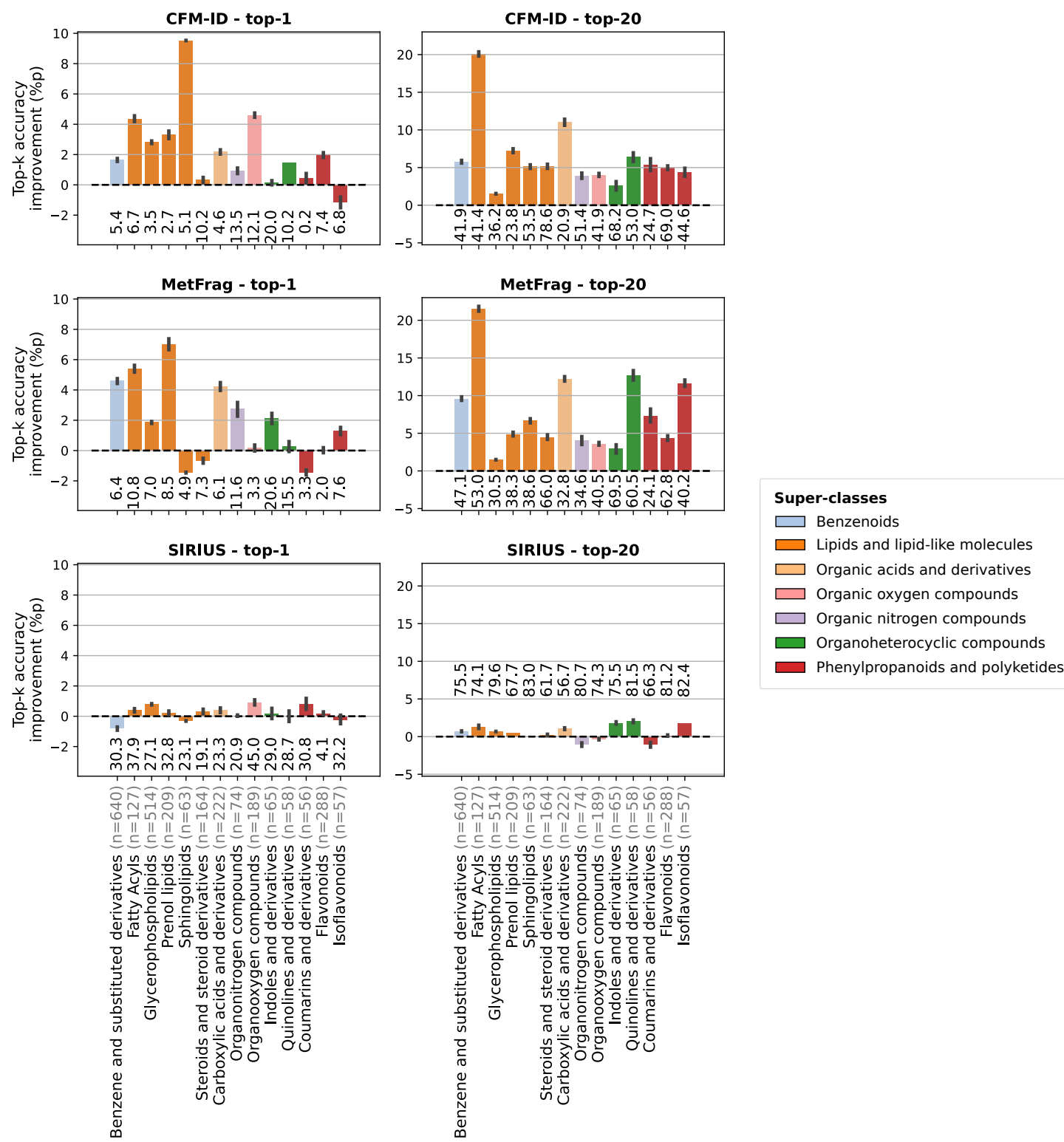

ClassyFire: Class

ClassyFire: Class

Figure 6: Performance gain by LC-MS ${ }^{2}$ Struct across ClassyFire class-level annotations. The figure shows the average and 95\%-confidence interval of the ranking performance (top-k) improvement of LC-MS ${ }^{2}$ Struct compared to Only-MS ${ }^{2}$ (baseline). The top-k accuracies (\%) under the bars show the Only-MS ${ }^{2}$ performance. For each molecule class, the number of unique molecular structures in the class is denoted in the $\mathrm{x}$-axis label (n). 

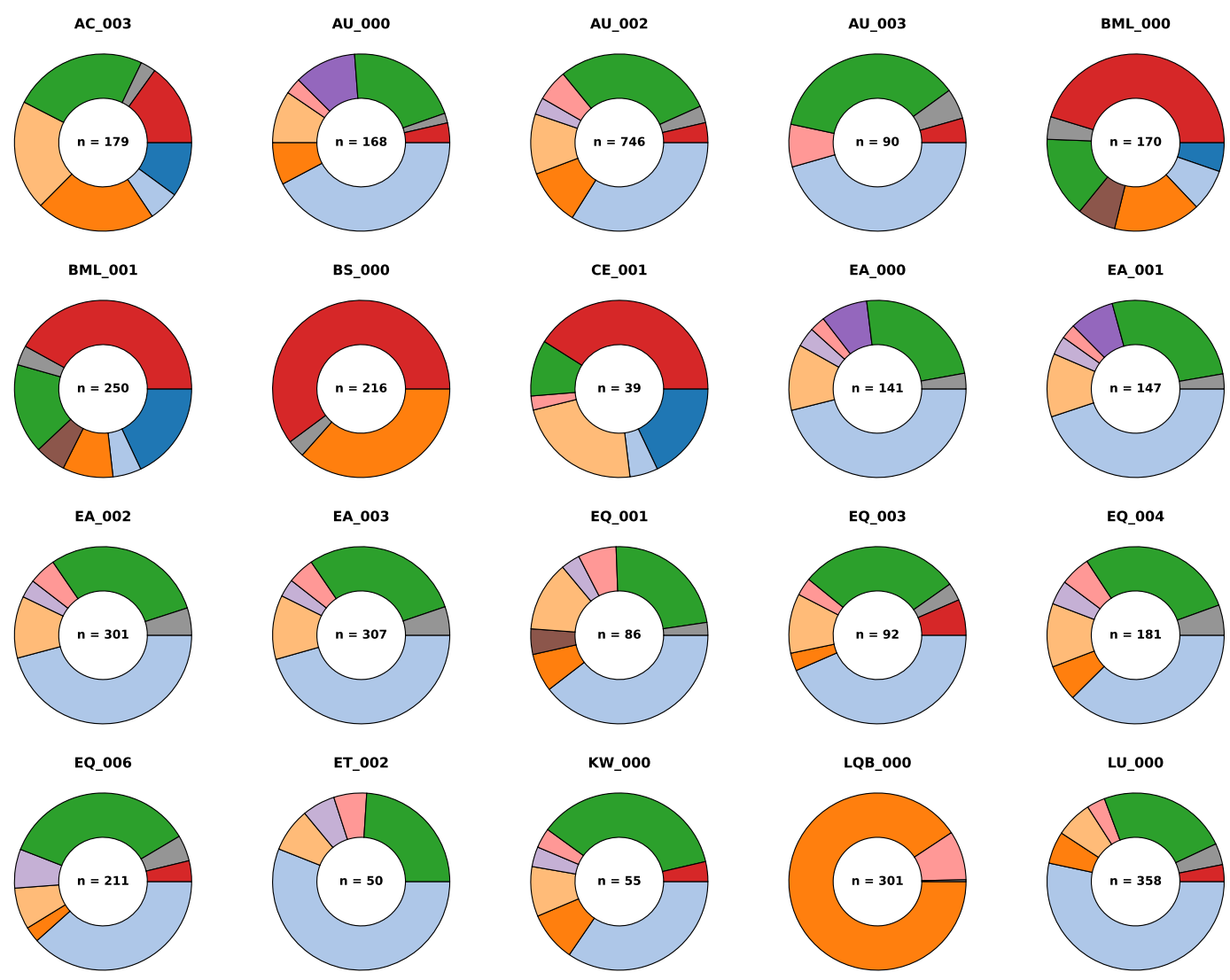

LU_001
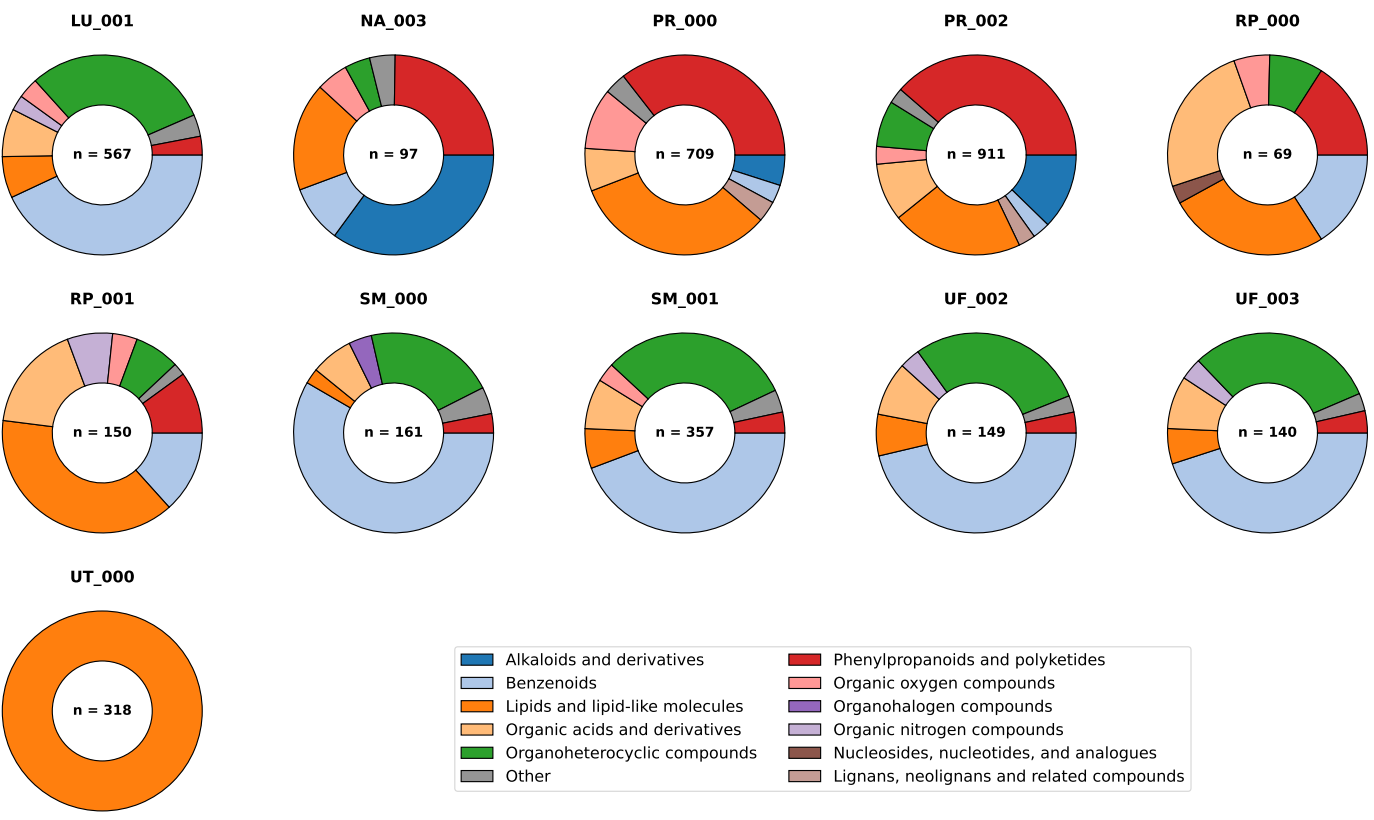

Figure 7: Distribution of molecule classes in the MassBank (MB) subsets. ClassyFire super-class distribution 51] for each MB-subset studied in our experiments. Within each MBsubset, the label "Other" is assigned to each super-class which makes up less then $2.5 \%$ of all molecules. The center label represents the number of examples for the respective MB-subset. 
bioRxiv preprint doi: https://doi.org/10.1101/2022.02.11.480137; this version posted February 14, 2022. The copyright holder for this preprint (which was not certified by peer review) is the author/funder, who has granted bioRxiv a license to display the preprint in perpetuity. It is made available under aCC-BY-NC-ND 4.0 International license.

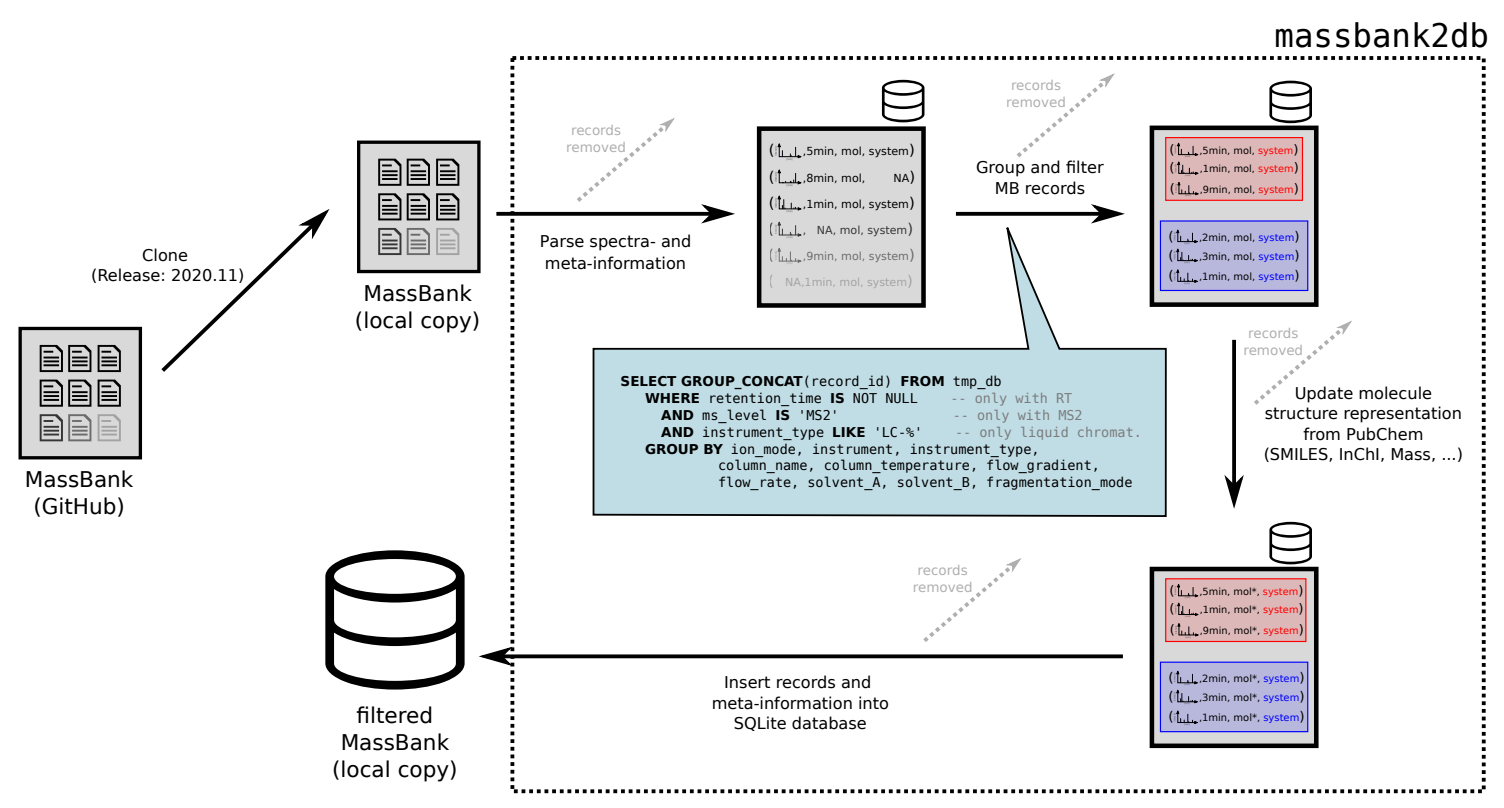

Figure 8: Processing pipeline of the MassBank data. Illustration of the processing pipeline to extract the training data from MassBank. The depicted workflow is implemented in the "massbank2db" Python package 47 . 
Table 1: Training and evaluation dataset sizes in our experiments. We provide the number $(\#)$ of $\left(\mathrm{MS}^{2}, \mathrm{RT}\right)$-tuples used for the generation of training and evaluation LC-MS ${ }^{2}$ experiments. For the ALLDATA setup the training and evaluation tuple-set is equal. The number of evaluation LC-MS ${ }^{2}$ experiments depends on the number of available evaluation tuples.

\begin{tabular}{|c|c|c|c|c|c|}
\hline \multirow[b]{2}{*}{ MB-subset } & \multicolumn{2}{|c|}{ ALLDATA } & \multicolumn{3}{|c|}{ ONLYSTEREO } \\
\hline & \#Tuples & \#Exp. & \#Tuples (train.) & \#Tuples (eval.) & \#Exp. \\
\hline AC_003 & 179 & 15 & 172 & 157 & 15 \\
\hline AU_000 & 168 & 15 & 146 & 23 & - \\
\hline AU_002 & 746 & 14 & 578 & 172 & 15 \\
\hline AU_003 & 90 & 15 & 77 & 21 & - \\
\hline BML_000 & 170 & 15 & 77 & 24 & - \\
\hline BML_001 & 250 & 15 & 125 & 33 & 1 \\
\hline BS_000 & 216 & 15 & 205 & 135 & 15 \\
\hline CE_001 & 39 & 1 & 30 & 19 & - \\
\hline EA_000 & 141 & 15 & 118 & 19 & - \\
\hline EA_001 & 147 & 15 & 126 & 19 & - \\
\hline EA_002 & 301 & 6 & 240 & 56 & 1 \\
\hline EA_003 & 307 & 6 & 246 & 57 & 1 \\
\hline EQ_001 & 86 & 15 & 68 & 28 & - \\
\hline EQ_003 & 92 & 15 & 64 & 6 & - \\
\hline EQ_004 & 181 & 15 & 127 & 51 & 1 \\
\hline EQ_006 & 211 & 15 & 138 & 15 & - \\
\hline ET_002 & 50 & 1 & 29 & 2 & - \\
\hline KW_000 & 55 & 1 & 43 & 4 & - \\
\hline LQB_000 & 301 & 6 & 271 & 270 & 5 \\
\hline LU_000 & 358 & 7 & 311 & 50 & 1 \\
\hline LU_001 & 567 & 11 & 472 & 101 & 15 \\
\hline NA_003 & 97 & 15 & 91 & 73 & 1 \\
\hline PR_000 & 709 & 14 & 131 & 21 & - \\
\hline PR_002 & 911 & 18 & 391 & 250 & 15 \\
\hline RP_000 & 69 & 1 & 55 & 35 & 1 \\
\hline RP_001 & 150 & 15 & 119 & 73 & 1 \\
\hline SM_000 & 161 & 15 & 136 & 12 & - \\
\hline SM_001 & 357 & 7 & 280 & 30 & 1 \\
\hline UF_002 & 149 & 15 & 124 & 18 & - \\
\hline UF_003 & 140 & 15 & 115 & 15 & - \\
\hline UT_000 & 318 & 6 & 294 & 293 & 5 \\
\hline Total & 7716 & 354 & 5399 & 2082 & 94 \\
\hline
\end{tabular}


Table 2: Median candidate set size for the MassBank (MB) subsets. The table shows the median number of molecular candidates per MB-subset used in our experiments. In the ALLDATA setup the candidates are identified by their InChIKey first block, where as for the Only-MS ${ }^{2}$ setup the full InChIKey is used. The candidate number is computed based on the MB records which are used in the simulated LC-MS ${ }^{2}$ experiments. For ONLYSTEREO, some MB-subsets are not used in the evaluation, and therefore their candidate set size is omitted (-).

\begin{tabular}{lrr}
\hline MB-subset & ALLDATA & ONLYSTEREO \\
\hline AC_003 & 305 & 384 \\
AU_000 & 269 & - \\
AU_002 & 1018.5 & 1434.5 \\
AU_003 & 1297 & - \\
BML_000 & 689 & - \\
BML_001 & 1013.5 & 1688 \\
BS_000 & 429 & 258 \\
CE_001 & 819 & - \\
EA_000 & 771 & - \\
EA_001 & 570 & - \\
EA_002 & 1373 & 1239 \\
EA_003 & 1306 & 1097 \\
EQ_001 & 425 & - \\
EQ_003 & 759.5 & - \\
EQ_004 & 872 & 1027 \\
EQ_006 & 1045 & - \\
ET_002 & 4957 & - \\
KW_000 & 2010 & - \\
LQB_000 & 73 & 106 \\
LU_000 & 533 & 362.5 \\
LU_001 & 998 & 751 \\
NA_003 & 1024 & 1608 \\
PR_000 & 109 & - \\
PR_002 & 228 & 636 \\
RP_000 & 760 & 1015 \\
RP_001 & 658 & 723 \\
SM_000 & 312 & - \\
SM_001 & 800 & 1095.5 \\
UF_002 & 1498 & - \\
UF_003 & 1392.5 & - \\
UT_000 & 56 & 93 \\
\hline
\end{tabular}


Table 3: MassBank (MB) information used to group the records. Two MassBank records are considered to belong to the same MB-subset in our experiments, if all properties listed in the table are equal between them. See https://github.com/MassBank/MassBank-web/blob/ main/Documentation/MassBankRecordFormat.md for a more comprehensive description of the MassBank records' fields.

\begin{tabular}{|c|c|c|}
\hline Property & Description & Example \\
\hline contributor & $\begin{array}{l}\text { Contributor who uploaded a } \\
\text { MassBank record }\end{array}$ & BGC_Munich \\
\hline accession prefix & $\begin{array}{l}2-3 \text { character long prefix further } \\
\text { specifying the records of a contrib- } \\
\text { utor }\end{array}$ & EA, EQ \\
\hline instrument_type & $\begin{array}{l}\text { General type of instrument used } \\
\text { for the LC-MS analysis }\end{array}$ & LC-ESI-QTOF \\
\hline ion_mode & MS Ionization mode & negative \\
\hline instrument & $\begin{array}{l}\text { Commercial name and manufac- } \\
\text { turer of the MS instrument }\end{array}$ & Bruker maXis Impact \\
\hline fragmentation_mode & $\begin{array}{l}\text { Fragmentation method used for } \\
\text { dissociation or fragmentation }\end{array}$ & CID \\
\hline column_name & $\begin{array}{l}\text { Commercial name and manufac- } \\
\text { turer of the LC instrument }\end{array}$ & Symmetry C18 Column, Waters \\
\hline column_temperature & $\begin{array}{l}\text { Static column temperature in LC- } \\
\text { MS }\end{array}$ & $40 \mathrm{C}$ \\
\hline flow_gradient & $\begin{array}{l}\text { Gradient of mobile phases in LC- } \\
\text { MS }\end{array}$ & $\begin{array}{l}0 \mathrm{~min}: 5 \%, \quad 24 \mathrm{~min}: 95 \% \quad \text { (acetoni- } \\
\text { trile) }\end{array}$ \\
\hline flow_rate & Flow Rate of liquid phase in LC & $300 \mathrm{uL} / \mathrm{min}$ \\
\hline solvent_A & $\begin{array}{l}\text { Chemical composition of buffer so- } \\
\text { lution }(\mathrm{A})\end{array}$ & $\mathrm{H} 2 \mathrm{O}(0.1 \% \mathrm{HCOOH})$ \\
\hline solvent_B & $\begin{array}{l}\text { Chemical composition of buffer so- } \\
\text { lution (B) }\end{array}$ & $\mathrm{CH} 3 \mathrm{CN}(0.1 \% \mathrm{HCOOH})$ \\
\hline
\end{tabular}




\section{${ }_{823}$ Supplementary material}

Table 4: Meta-information for the MassBank (MB) subsets. The the LC- and MSconditions for each MB-subset.

THIS TABLE IS PROVIDED IN A SEPARATE FILE: massbank_groups_meta_data.tsv 